\title{
Molecular Dynamics Studies of Load Transfer in Nanocomposites Reinforced by Defective Carbon Nanotube
}

by

Xudong Peng, B.Eng.

A thesis submitted in conformity with the requirements for the degree of Master of Applied Science

Department of Mechanical and Industrial Engineering University of Toronto

(C) Copyright by Xudong Peng 2016 


\title{
Molecular Dynamics Studies of Load Transfer in Nanocomposites Reinforced by Defective Carbon Nanotube
}

\author{
Xudong Peng \\ Master of Applied Science \\ Department of Mechanical and Industrial Engineering \\ University of Toronto
}

2016

\begin{abstract}
This study is concerned with the development of relatively more accurate numerical simulations than those adopted in the literature to investigate the considerable discrepancies between experimental findings and theoretical predictions of the interfacial shear strength (ISS) of nanoreinforced thermoset composites. In this work, extensive molecular dynamics (MD) simulations were conducted to examine the ISS and buckling behavior of carbon nanotube (CNT)-reinforced epoxy composites. We considered different types of defects such as vacancy, Stone-Wales defect, carbon adatom, and phenyl functional group. Pull-out and compressive load simulations were performed via the consistent valence forcefield (CVFF) on a representative volume element comprising a single-walled CNT embedded in an epoxy matrix. Our results revealed that different defects can, to some extent, either enhance or degrade the properties of nanocomposites. The findings will assist in improving our understanding of the toughening/weakening mechanisms associated with nanoscopic reinforcement and the load transfer capability in epoxy-based nanocomposites.
\end{abstract}




\section{Acknowledgments}

I would like to offer my greatest appreciation and gratitude to Prof. Shaker Meguid for his expert guidance, financial assistance and continued support throughout the course of my research. I also wish to appreciate all the past and present members of the Mechanics and Aerospace Design Laboratory for their friendship and technical assistance. Furthermore, I would like to acknowledge the financial support of the National Science and Engineering Research Council of Canada, the Discovery Accelerator Supplement, and the Mechanical and Industrial Engineering Fellowship gratefully. Finally, this thesis is dedicated to my parents and friends for their continued support and understanding. 


\section{Table of Contents}

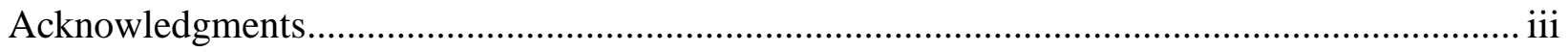

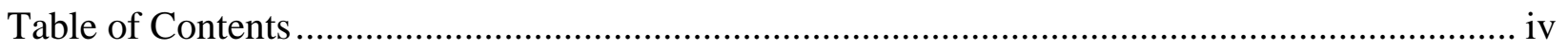

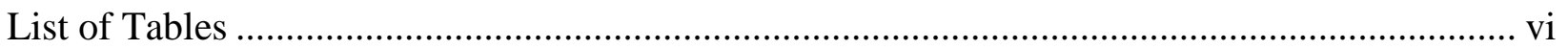

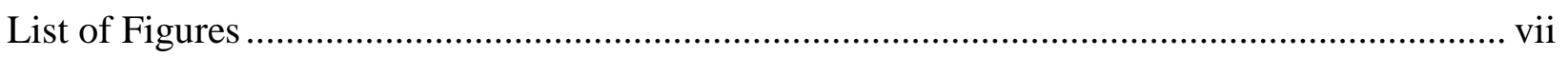

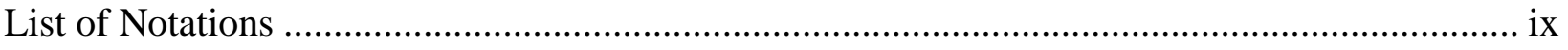

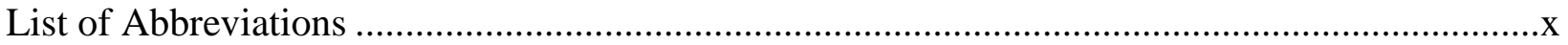

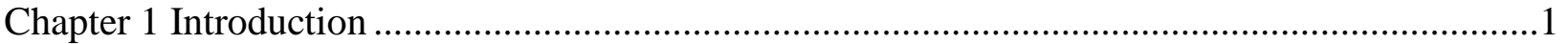

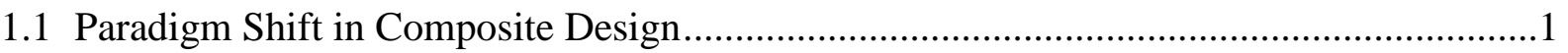

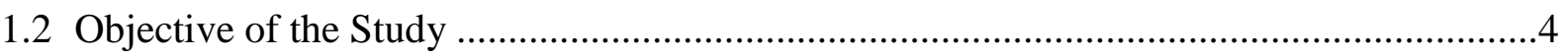

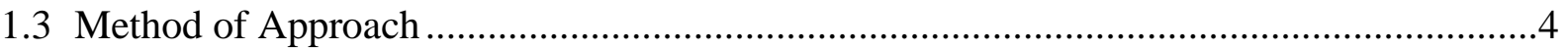

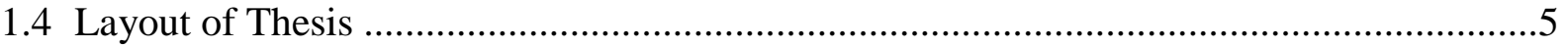

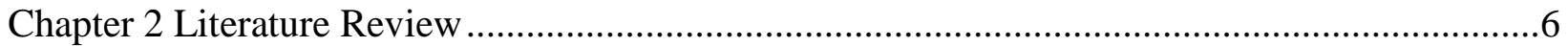

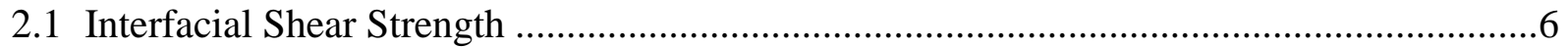

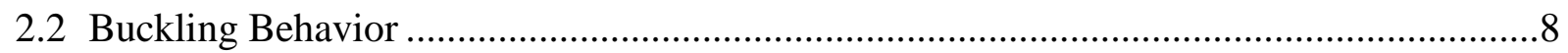

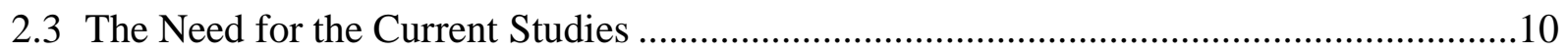

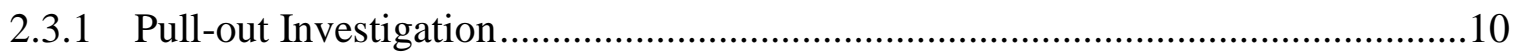

2.3.2 Compressive Load Investigation.....................................................................11

Chapter 3 Fundamental Aspects of MD Simulation Techniques.................................................13

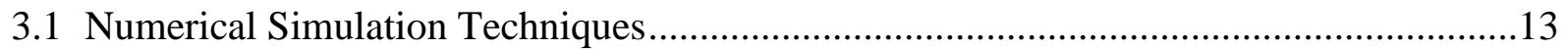

3.2 Molecular Modeling of Pull-out Simulation...............................................................14

3.2.1 Molecular Structure of CNTs with Defects and Functionalization .......................14

3.2.2 Cured versus Uncured Polymer ........................................................................16

3.2.3 Construction of Nanocomposite RVE ............................................................17

3.3 Molecular Modeling of Compressive Load Simulation......................................................18

3.3.1 Molecular Structure of CNTs with Defects ........................................................18

3.3.2 Construction of Freestanding CNT and Nanocomposite RVE .............................19

Chapter 4 Molecular Dynamics Simulations ..............................................................................2

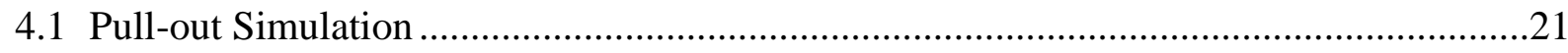

4.1.1 CNT Pull-out Method ......................................................................................21

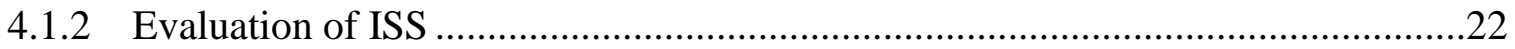

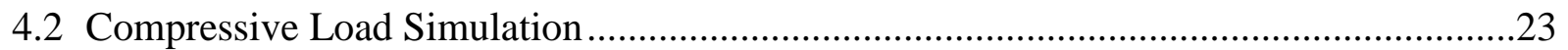

4.2.1 CNT and RVE Compressive Load Method ......................................................23 


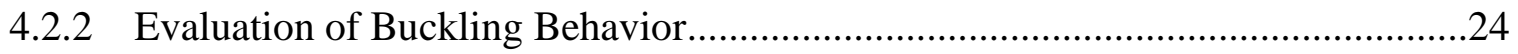

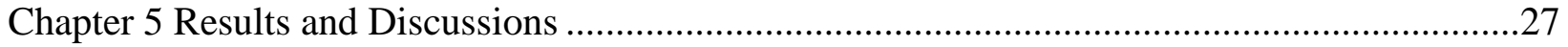

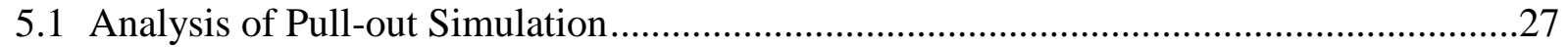

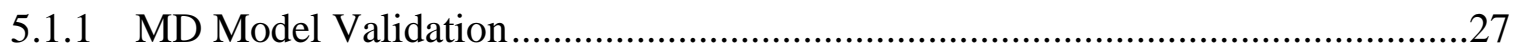

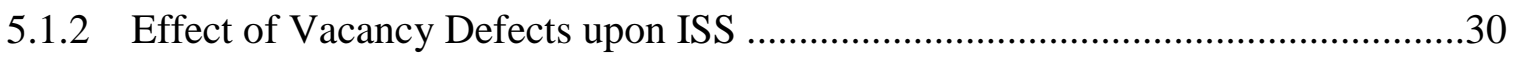

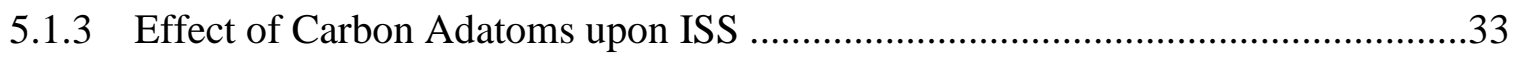

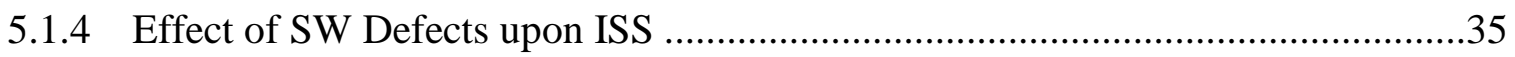

5.1.5 Effect of Phenyl Functional Groups upon ISS .................................................37

5.2 Analysis of Compressive Load Simulation................................................................40

5.2.1 Effect of Vacancy Defects upon freestanding SWCNTs ......................................40

5.2.2 Effect of Missing Atoms upon Embedded SWCNTs .......................................43

5.2.3 Effect of Vacancy Symmetry and Distribution upon Embedded SWCNTs .........47

5.2.4 Effect of SW defects upon Freestanding and Embedded SWCNTs ....................47

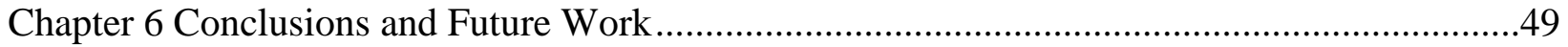

6.1 General Conclusions of Pull-out Simulation ................................................................49

6.2 General Conclusions of Compressive Load Simulation ...........................................50

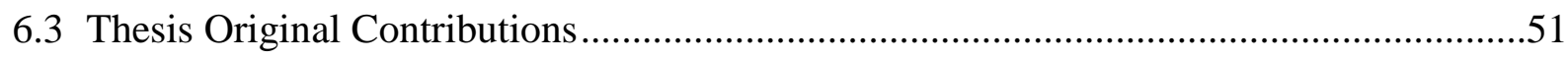

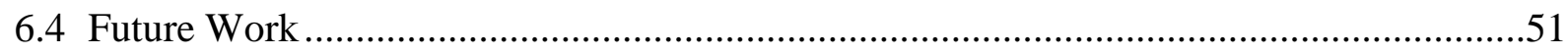

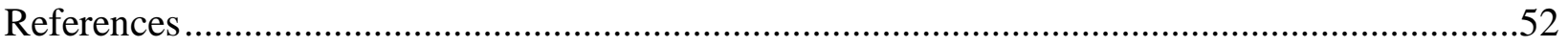




\section{List of Tables}

Table 4.1 Composition of uncured and cured nanocomposite RVEs immediately before pristine CNT pull-out simulation.

Table 4.2 Composition of freestanding SWCNTs and RVEs containing different SWCNTs immediately before compression simulations...

Table 5.1 Comparison of buckling behavior of freestanding defective SWCNTs against the buckling behavior of freestanding pristine SWCNTs.

Table 5.2 Comparison of buckling behavior of embedded defective SWCNTs against the buckling behavior of embedded pristine SWCNTs. 


\section{List of Figures}

Fig. 1.1 Commonly observed CNT-reinforced composite toughening mechanisms (a) CNT bridging, (b) crack deflection, and (c) covalent bonding at CNT-matrix interface. ..................... 3

Fig. 1.2 Commonly observed CNT-reinforced composite weakening mechanisms (a) single vacancy, (b) Stone-Wales defect, (c) carbon external atom, (d) CNT buckling, (e) CNT

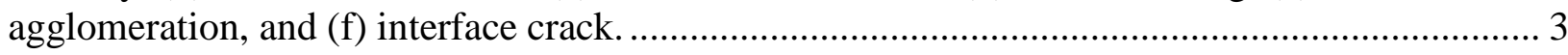

Fig. 1.3 Formation of RVE of CNT-reinforced composites. .................................................. 4

Fig. 1.4 Research method of this study............................................................................ 5

Fig. 3.1 Schematics of different bonded CVFF terms: (a) bond length, (b) bond angle, (c) torsion

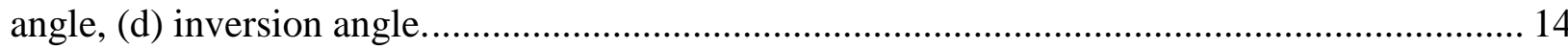

Fig. 3.2 Geometries of CNTs containing different defects and functionalization: (a) monovacancy, (b) bivacancy, (c) trivacancy, (d) single carbon adatom defect, (e) SW defect, and (f) phenyl group functionalization.

Fig. 3.3 Chemical structures of (a) epoxy resin (DGEBA), (b) curing agent (TETA), and (c) cured epoxy oligomer (6 DGEBA molecules connected by 1 TETA).

Fig. 3.4 Preparation of the RVE (a) SWCNT embedded in 33 randomly placed cured epoxy oligomers in a simulation box of size $150 \AA$ X $150 \AA$ X $45 \AA$, and (b) the compressed SWCNTepoxy system in a simulation box of size $50 \AA$ X $50 \AA$ X $42 \AA$.

Fig. 3.5 Defects studied in this research ( $0 \mathrm{~A}$ - pristine, $1 \mathrm{~A}$ - monovacancy, 1B - symmetrically distributed two monovacancies, 1C - asymmetrically distributed two monovacancies, $2 \mathrm{~B}-$ symmetric bivacancy, 2C - asymmetric bivacancy, 3A - symmetric trivacancy, 3B - asymmetric trivacancy, $1 \mathrm{SW}-\mathrm{A}$ - symmetric SW defect, 1SW-B - asymmetric SW defect)..................... 19

Fig. 4.1 Simulation setup for the pull-out of a CNT from the epoxy matrix........................... 21

Fig. 4.2 Setup for the buckling simulations of (a) freestanding SWCNT, and (b) SWCNT embedded in the epoxy matrix. 24

Fig. 4.3 (a) Strain energy-strain curves; (b) force-displacement curves for different pristine freestanding and embedded SWCNTs.

Fig. 4.4 Buckling mode shapes of the freestanding SWCNTs (a) $(7,7)$, (b) $(9,9)$ and (c) $(12,0)$.

Fig. 5.1 Schematics of the pull-out processes of pristine CNT embedded in (a) uncured epoxy matrix and (b) cured epoxy matrix. 28

Fig. 5.2 Potential Energy variations of pristine CNT with pull-out distance in the uncured and cured epoxy composites.

Fig. 5.3 Spatially averaged concentration profiles versus distance from the center of the CNT before and after curing (a) for all atoms and (b) for $\mathrm{sp}^{2}$-hybridized carbon atoms.

Fig. 5.4 Aromatic ring distribution near the CNT in (a) uncured epoxy matrix and (b) cured epoxy matrix. 
Fig. 5.5 Variations in ISS with number of missing atoms prior to and post curing.

Fig. 5.6 Relative concentrations of $\mathrm{sp}^{2}$ carbon atoms for (a) different monovacancies after curing, (b) different bivacancies after curing, and (c) different trivacancies after curing.

Fig. 5.7 Variations in interfacial binding energy between vacancy defective CNTs and cured epoxy matrix with pull-out distance.

Fig. 5.8 Cross sections of the pull-out process for pristine and vacancy defective CNT in cured epoxy matrix at $\mathrm{z}=30 \AA$ A: (a) pristine, (b) 6 monovacancies, (c) 5 bivacancies, and (d) 4 trivacancies.

Fig. 5.9 Variations in ISS with number of adatom defects prior to and post curing. 34

Fig. 5.10 Relative concentrations of $\mathrm{sp}^{2}$ carbon atoms for different adatoms after curing. 34

Fig. 5.11 Variations in interfacial binding energy between adatom defective CNTs and cured epoxy matrix with pull-out distance. 35

Fig. 5.12 Cross section of the pull-out process for 12 adatom defective CNT in cured epoxy matrix at $\mathrm{z}=30 \AA$. 35

Fig. 5.13 Variations in ISS with number of SW defects prior to and post curing. .................... 36

Fig. 5.14 Relative concentrations of $\mathrm{sp}^{2}$ carbon atoms for different $\mathrm{SW}$ defects after curing..... 36

Fig. 5.15 Variations in interfacial binding energy between SW defective CNTs and cured epoxy matrix with pull-out distance. 37

Fig. 5.16 Cross section of the pull-out process for $15 \mathrm{SW}$ defective CNT in cured epoxy matrix at $\mathrm{z}=30 \AA$.

Fig. 5.17 Variations in ISS with number of phenyl groups prior to and post curing.................. 38

Fig. 5.18 Relative concentrations of $\mathrm{sp}^{2}$ carbon atoms for different phenyl groups after curing. 38

Fig. 5.19 Variations in interfacial binding energy between functionalized CNTs and cured epoxy matrix with pull-out distance.

Fig. 5.20 Cross section of the pull-out process for 9 phenyl group functionalized CNT in cured epoxy matrix the at $\mathrm{z}=30 \AA$.

Fig. 5.21 (a), (c) and (e) Strain energy-strain curves; (b), (d) and (f) force-displacement curves for different freestanding defective SWCNTs.

Fig. 5.22 Buckling mode shapes of the embedded SWCNTs (a), $(7,7)$, (b) $(9,9)$ and (c) $(12,0)$.

Fig. 5.23 (a), (c) and (e) Strain energy-strain curves; (b), (d) and (f) force-displacement curves for different embedded defective SWCNTs. 


\section{List of Notations}

A

$A_{c}$

C

E

$E_{\text {cnt }}$

$E_{\text {epoxy }}$

$E_{\text {interaction }}$

$E_{\text {potential }}$

$E_{\text {potential }}^{\text {initial }}$

$E_{\text {potential }}^{\text {final }}$

F

$\boldsymbol{F}_{i} \quad$ Force vector acting on atom i

$l$

$L \quad$ Original length of CNT

$\Delta L \quad$ End-shortening displacement

$m_{i} \quad$ Mass of ith atom

$N \quad$ Total number of atoms

$N_{\text {sub }} \quad$ Number of atoms in sub-

volume

$N_{\text {tot }} \quad$ Total number of atoms in

RVE
$P_{c r} \quad$ Critical buckling load

Position vector of an atom

Position vector of atom $\mathrm{i}$

Time

Potential energy of a system

Strain energy of CNT

Deformation energy of bond angles

$U_{\text {bond }} \quad$ Deformation energy of bond lengths

$U_{\text {Coulomb }} \quad$ Electrostatic interaction

$U_{\text {dihedral }}$ Deformation energy of torsion angles

$U_{\text {improper }} \quad$ Deformation energy of inversion angles

$U_{v d W} \quad$ Van der Waals interaction

$V_{\text {sub }} \quad$ Volume of sub-volume

$V_{\text {tot }} \quad$ Total volume of RVE

$W_{\text {pull-out }} \quad$ Pull-out work

$z$

Pull-out distance from initial position to final position of CNT

$\tau_{i} \quad$ Interfacial shear strength

$\gamma \quad$ Interfacial binding energy

$\varepsilon \quad$ Strain of CNT

$\varepsilon_{c r} \quad$ Critical buckling strain

$\Delta_{\mathrm{cr}} \quad$ Critical buckling displacement 


\section{List of Abbreviations}

$\begin{array}{llll}\text { 0A } & \text { Pristine } & \text { ISS } & \text { Interfacial shear strength } \\ \text { 1A } & \text { Monovacancy } & \text { LAMMPS } & \begin{array}{l}\text { Large-scale atomic/molecular } \\ \text { massively parallel simulator }\end{array} \\ \text { 1B } & \begin{array}{l}\text { Symmetrically distributed two } \\ \text { monovacancies }\end{array} & \text { LJ } & \text { Lennard-Jones } \\ \text { 1C } & \begin{array}{l}\text { Asymmetrically distributed } \\ \text { two monovacancies }\end{array} & \text { MD } & \text { Molecular dynamics } \\ \text { 2B } & \text { Symmetric bivacancy } & \text { MM } & \text { Molecular mechanics } \\ \text { 2C } & \text { Asymmetric bivacancy } & \text { MWCNT } & \text { Multi-walled CNT } \\ \text { 3A } & \text { Symmetric trivacancy } & \text { NPT } & \text { Isothermal-isobaric ensemble } \\ \text { 3B } & \text { Asymmetric trivacancy } & \text { NVT } & \text { Canonical ensemble } \\ \text { 1SW-A } & \text { Symmetric SW defect } & \text { PE } & \text { Polyethylene } \\ \text { 1SW-B } & \text { Asymmetric SW defect } & \text { RVE } & \text { Representative volume element } \\ \text { AFM } & \text { Atomic force microscope } & \text { SW } & \text { Stone-Wales } \\ \text { CFRP } & \text { Carbon fiber reinforced plastic } & \text { SWCNT } & \text { Single-walled CNT } \\ \text { CNT } & \text { Carbon nanotube } & \text { TEM } & \text { Transmission electron } \\ \text { CVFF } & \text { Consistent valence forcefield } & \text { TETA } & \text { Triethylene tetramine } \\ \text { DGEBA } & \text { Diglycidyl ether of bisphenol } & \text { vdW } & \text { Van der Waals } \\ & \text { A } & & \\ \text { GLARE } & \text { Glass laminate aluminum } & & \\ & \text { reinforced epoxy } & & \end{array}$




\section{Chapter 1 \\ Introduction}

Summary: In this chapter, we define the problem, justify the undertaking of the study and outline the method of approach adopted in achieving the research objectives. Furthermore, we provide a summary of the layout of the thesis.

\subsection{Paradigm Shift in Composite Design}

Composite materials are composed of two or more constituents with significantly different mechanical, chemical and/or physical properties that, when combined, they attain superior properties when compared to the properties of individual components. One of the leading examples of incorporating advanced composite materials into reality is the use of GLARE or "Glass Laminate Aluminum Reinforced Epoxy" in Airbus A380. GLARE is a metal-fiber laminate made from alternating sheets of glass-epoxy prepreg and layers of aluminum [1], which makes up 22\% of the primary structures in Airbus A380 and offers a weight savings of between 15 and 30\% over aluminum alloy [2]. Another interesting aerospace application is Boeing 787 (Dreamliner), which contains some $50 \%$ of carbon fiber reinforced plastic (CFRP) [3]. This translates into an average weight savings of $20 \%$ [4]. Apart from the obvious fact that lighter aircraft leads to less fuel consumption, these composite materials are also preferred for their high strength, corrosion resistance, enhanced damage tolerance and improved fatigue resistance when compared to traditional aluminum and steel. However, such composites suffer from a number of disadvantages. For example, it is hard to produce evenly dispersed CFRPs, which significantly affects their resulting mechanical properties. Another drawback is the fact that reinforcing composites by glass or carbon fibers with a finite size leads to vacancies/cracks at the bond interface[5]. Hence, the development of advanced composites has begun to shift from traditional composites to nanocomposites, taking advantage of the unique combination of mechanical and physical properties of nanofillers (fillers with a characteristic dimension below $100 \mathrm{~nm}$ ). There are various benefits related to dispersing nanofillers in polymeric materials. While some credit is a result of the intrinsic properties of the fillers, most benefits stem from a significant reduction in filler size together with a substantial enhancement in the interfacial area between the fillers and matrix phases. Therefore, the dispersion of just a few volume percentages of nanofillers into 
polymeric matrices could result in dramatic changes in their mechanical properties with added functionalities.

In this work, we focus on one of the most popular nanofillers: carbon nanotubes (CNTs). Since their discovery by Iijima [6], CNTs have attracted considerable interest from the mechanics and materials communities. This is because of their potential as reinforcements in polymer-based nanocomposites. Compared with traditional materials, such newly advanced polymer nanocomposites can provide superior multifunctional properties including high mechanical, electrical, and thermal properties [7]. In particular, the focus of the current work is to study the mechanical interface properties of CNT-reinforced polymer composites. The mechanical properties of CNT-reinforced composites are significantly influenced by the interface condition (cohesion and adhesion) between the CNT and the surrounding matrix. Higher interfacial shear strength (ISS) is an indicator of better stress transfer from the polymer matrix to the embedded nanotube, and hence the enhanced reinforcement's effect. Furthermore, there are three typical toughening mechanisms associated with nanoscopic reinforcement. The first shown in Fig. 1.1(a) is crack bridging where a CNT acts as an obstacle for a propagating crack and assists in closing the crack. This nanotube bridge can only be overcome by fracturing the CNT or by shearing the interface, resulting in a dissipation of energy. Accordingly, the CNT could be pulled out entirely, pulled out in a sword-in-sheath fashion, or fractured depending on the characteristics of the CNT-composite interface. Crack pinning (shown in Fig. 1.1(b)) is the second mechanism where the propagating crack encounters a CNT and is forced to propagate around it. This leads to a greater increase in the crack path, resulting in a greater dissipation of fracture energy and improved life. As demonstrated in Fig. 1.1(c), the CNT-reinforced nanocomposites can also be strengthened by forming covalent bonding at CNT-matrix interface, which makes it harder to pull out the CNT from the matrix. As shown in Fig. 1.2, researchers have also reported some weakening mechanisms such as vacancy, Stone-Wales (SW) defect, interstitial atom, CNT buckling, CNT agglomeration, and interface cracks. To achieve the full potential of CNTreinforced composites, researchers have carried out many experimental, analytical, and numerical investigations to study the interfacial properties of CNT-reinforced thermoplastics and thermoset composites [8-13]. However, there exist significant discrepancies amongst the results in the literature. This is partly due to oversimplified numerical simulations caused by computational limitations and the lack of interfacial bonding and partly due to deficient 
theoretical and numerical models that are either grossly oversimplified or inaccurate. Moreover, very limited works have been reported on the effect of buckling, external atom, and defects on the interfacial properties of CNT-reinforced composites. It is these limitations that have prompted the undertaking of the current study.

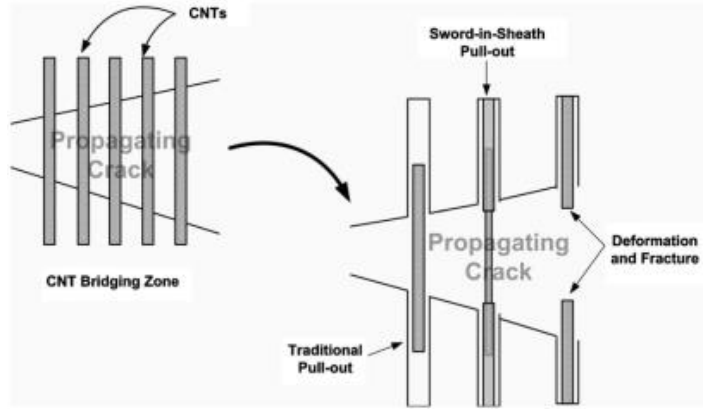

(a)

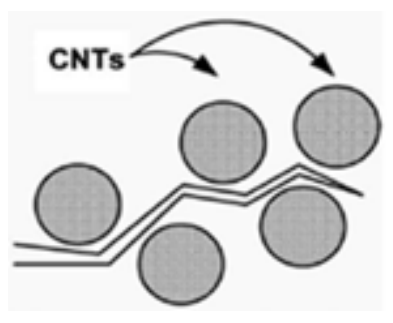

(b)

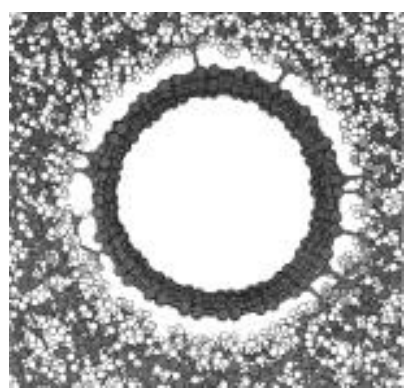

(c)

Fig. 1.1 Commonly observed CNT-reinforced composite toughening mechanisms (a) CNT bridging (from Ref. [14]), (b) crack deflection (from Ref. [14]), and (c) covalent bonding at CNT-matrix interface (from Ref. [15]).

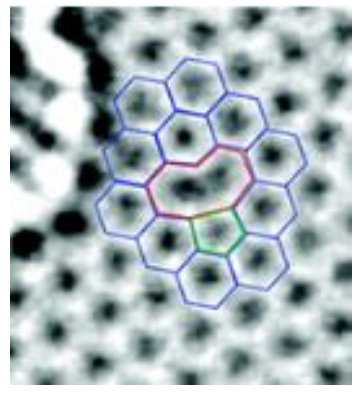

(a)

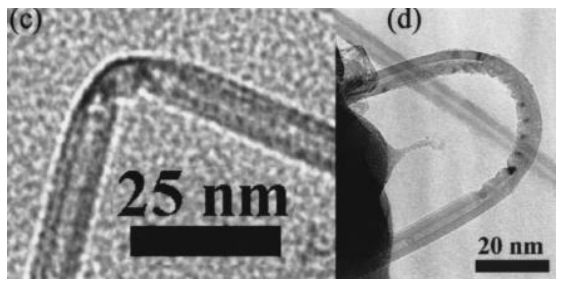

(d)

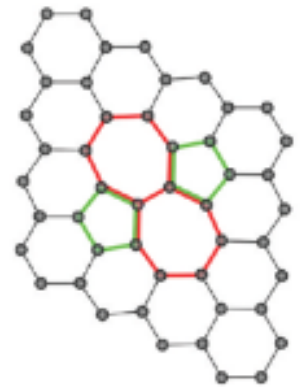

(b)

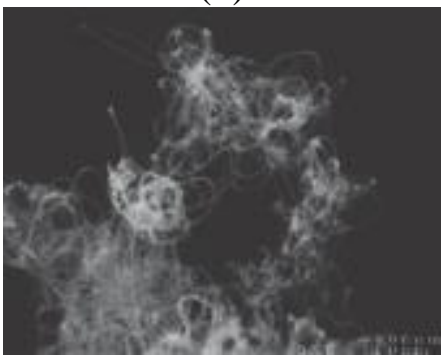

(e)

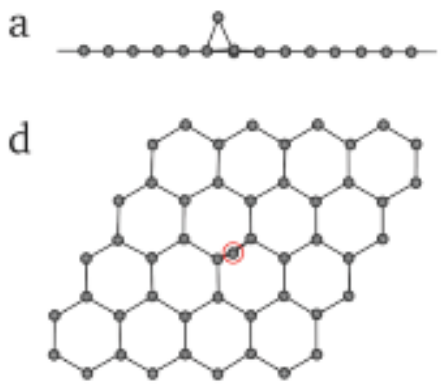

(c)

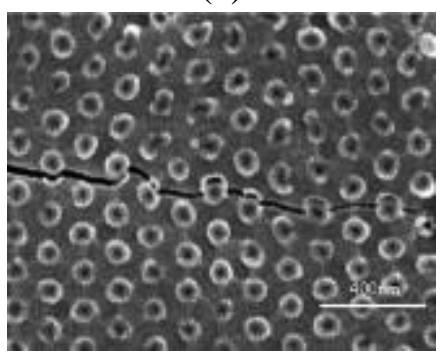

(f)

Fig. 1.2 Commonly observed CNT-reinforced composite weakening mechanisms (a) single vacancy (from Ref. [16]), (b) Stone-Wales defect (from Ref. [17]), (c) carbon external atom (from Ref. [17]), (d) CNT buckling (from Ref. [18]), (e) CNT agglomeration (from Ref. [19]), and (f) interface crack (from Ref. [20]). 


\subsection{Objective of the Study}

It is, therefore, the objective of this research is to:

(i) Evaluate the interfacial shear strength of CNT-reinforced thermoset composites and examine the effect of the following parameters on the strength of the interface:
a. Defects in embedded CNTs
b. Presence of foreign atoms
c. Interfacial non-bonded (van der Waals) and bonded (covalent bonding) interactions

d. Buckling of embedded CNTs

(ii) Investigate which of these parameters either singly or jointly have major effect on the interfacial shear strength of CNT-reinforced composites.

\subsection{Method of Approach}

In this research, we focus our efforts on investigating the effect of various parameters on the ISS of CNT-reinforced composites. Due to the difficulties of setting up nanoscale experiments and unavailability of high precision measuring instruments, we rely on molecular dynamics (MD) simulation, which is the most popular atomistic-based method for studying nanoscale behavior, to carry out our studies. CNT pull-out and compressive load tests have been well recognized as standard methods for evaluating the mechanical properties of nanocomposites. It is with this in mind that we conduct numerous numerical tests to investigate the set objectives. As shown in Fig. 1.3, a representative volume element (RVE) is then taken as a simulation box consisting of a $\mathrm{CNT}$ embedded at the center of matrix molecules.

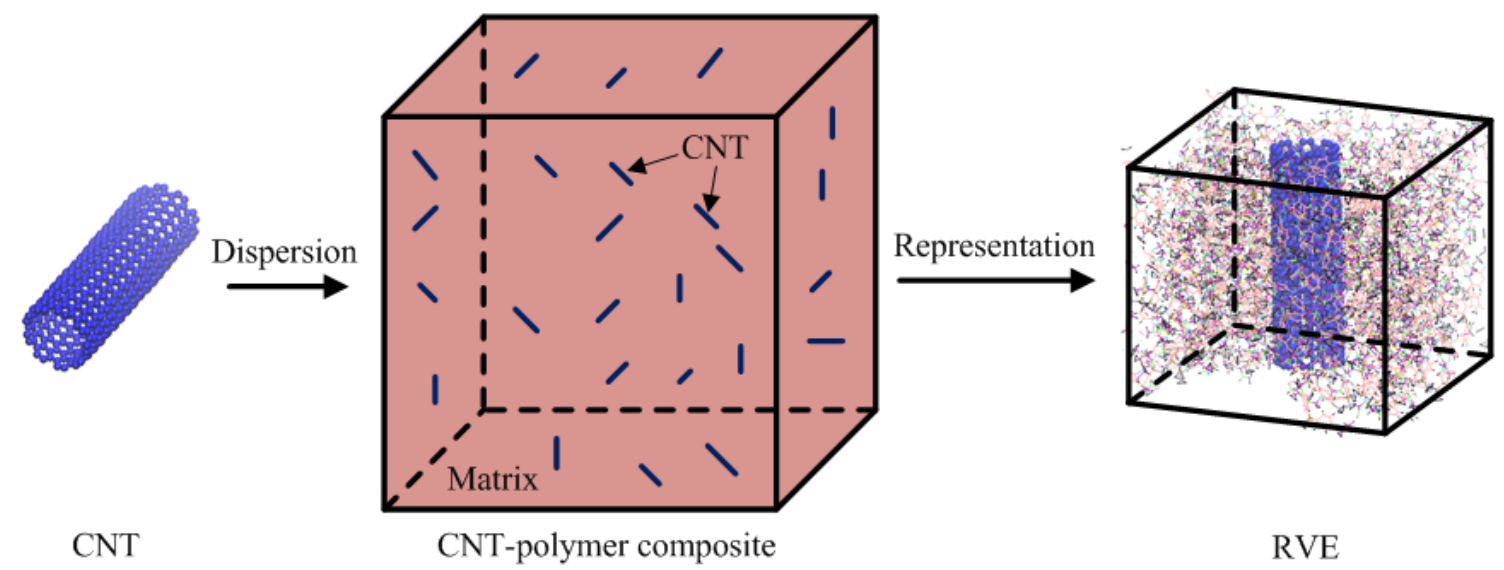

Fig. 1.3 Formation of RVE of CNT-reinforced composites. 
Since both numerical pull-out and buckling tests are considered, we decompose the research program into both pull-out modeling and compressive load modeling (shown in Fig. 1.4). The aims of the pull-out modeling are to explore the effect of defects, foreign atoms, and covalent bonding on the interfacial properties of nanocomposites. Different types of defects such as monovacancy, bivacancy, trivacancy, and SW defect are investigated, and their ISS is compared with the ISS for pristine CNTs. We further examined the effects of defects on the compressive behavior of CNTs with emphases on their buckling behavior, as this will affect the effective bulk properties of the nanocomposite. We first examine the effect of defects on the buckling behavior of freestanding CNTs. Different defect configurations and distributions are considered. This is followed by repeating the numerical experiments with defective CNTs embedded in a matrix and comparing their buckling results with the predictions of freestanding CNTs to identify the matrix effect.

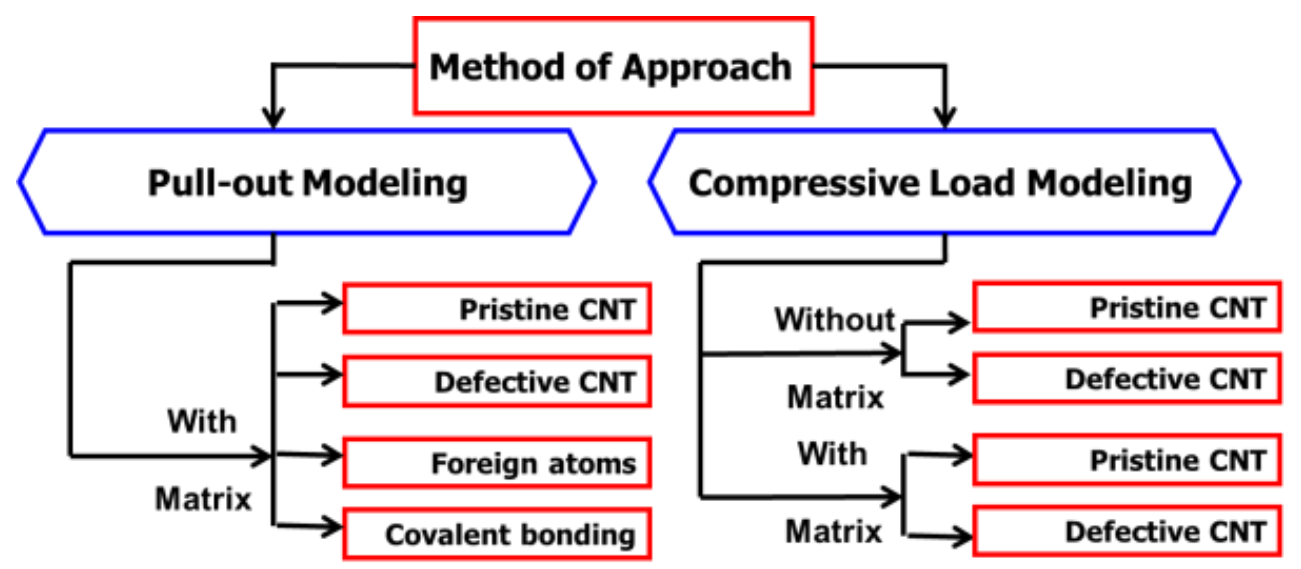

Fig. 1.4 Research method of this study.

\subsection{Layout of Thesis}

This thesis is divided into six chapters. After this introduction, Chapter 2 provides a critical review of the relevant investigations on the interfacial properties and buckling behavior of CNTreinforced composites. Chapter 3 offers details of the simulation techniques, followed by molecular modeling preparations for studying pull-out and compressive load simulations. Chapter 4 describes the pull-out and compressive load methods for conducting MD simulations as well as theories for analyzing their results. Chapter 5 discusses the results of parametrical studies obtained through MD simulations. Finally, in Chapter 6 we conclude the research and identify the original contributions of the thesis and provide suggestions for related future work. 


\section{Chapter 2 Literature Review}

Summary: This literature review is divided into three main sections. The first addresses experimental, analytical, and numerical studies of the ISS of CNT-reinforced composites. The second deals with the buckling investigations of freestanding and embedded CNTs. Finally, in the last section, we identify the need for the current studies.

\subsection{Interfacial Shear Strength}

Numerous experimental efforts have been devoted to the study of the ISS of CNT-reinforced composites. For example, Cooper et al. [21] calculated the ISS for single-walled CNTs (SWCNTs) and multi-walled CNTs (MWCNTs) embedded in an epoxy matrix based on pull-out experiments using a scanning probe microscope. In both cases, the ISS was found to be in the range of 35 to $376 \mathrm{MPa}$. Barber et al. [22] employed an atomic force microscope (AFM) to carry out direct CNT pull-out tests. They observed an average ISS of $47 \mathrm{MPa}$ for MWCNTs embedded in the polyethylene (PE)-butene matrix. Barber et al. [23] further investigated the effect of functionalization using both pristine and chemically modified MWCNTs in their AFM experiments. They found that for different embedded length, the measured ISS ranges from 10 MPa to $50 \mathrm{MPa}$ for pristine SWCNTs and from $20 \mathrm{MPa}$ to $165 \mathrm{MPa}$ for MWCNTs. Qian et al. [24] conducted tensile tests on composite films containing MWCNTs and polystyrene matrices. They found that the addition of $1 \mathrm{wt} \%$ CNTs leads to $36 \%-42 \%$ and $\sim 25 \%$ improvements in the elastic modulus and fracture stress, respectively. Meguid and Sun [25] also reported that the homogeneous dispersion of CNTs in an epoxy adhesive can improve the bonding and shear properties of composite interfaces by 1.2 times and 1.3 times the pure epoxy adhesive. Xu et al. [26] added $0.1 \mathrm{wt} \%$ MWCNTs into thin epoxy films and observed a $20 \%$ increase in the elastic

modulus using a shaft-loaded blister test. The ISS was estimated from 88 to $280 \mathrm{MPa}$ based on transmission electron microscopy (TEM) images.

Several analytical works have also been conducted to shed light on the complexities and the marked discrepancies in the reported ISS of CNT-polymer composites. For instance, Wagner et al. [27] combined fragmentation tests with the Kelly-Tyson model modified for hollow tubes to estimate the efficiency and quality of CNT-urethane composite interfaces. The ISS was obtained to be on the order of $500 \mathrm{MPa}$ and higher. Xu et al. [26] further applied the same modified Kelly- 
Tyson model to calculate the ISS theoretically and found it to be in the range of $88 \mathrm{MPa}$ to 280 MPa for different diameters MWCNTs. Gao and Li [28] modified the shear-lag model originally proposed by Cox [29] and used a cylindrical matrix embedded with a capped SWCNT as the RVE of a nano-reinforced polymer composite. The maximum ISS for different aspect ratios of the RVE was predicted to be about twice as large as the axial normal stress in the matrix. Li et al. [30] employed a refined micromechanics model including three concentric cylinders as the RVE for assessing the ISS in CNT-reinforced polymer composites. They reported that the ISS normalized by the axial normal stress in the matrix can range from 0.2 to 1.5 depending on the different volume fractions, aspect ratios, and end gaps.

In addition to existing experimental and analytical investigations, numerous numerical simulations have also been conducted to study the interfacial behavior of CNT-reinforced composites. For instance, Gou et al. [31, 32] investigated the ISS of individual SWCNTs and CNT ropes via both molecular mechanics (MM) and molecular dynamics (MD) simulations. The ISS was calculated to be $61 \mathrm{MPa}$ and $36 \mathrm{MPa}$ for a SWCNT and a three CNT rope embedded in an epoxy matrix, respectively. Liu et al. [33] used MD simulations to investigate the influence of noncovalent and covalent functionalization on the interfacial properties of SWCNT-epoxy system. They found that properly designed hybrid system containing both covalent and noncovalent functionalization can increase the ISS to $940 \mathrm{MPa}$ from $170 \mathrm{MPa}$ for a pristine SWCNT-epoxy system. Li et al. [34] further conducted pull-out simulations on SWCNT-PE systems. Instead of using conventional uniform distribution of ISS on the entire sidewalls of CNTs, they assumed that the ISS was distributed uniformly within $1 \mathrm{~nm}$ from each end of the CNTs but was zero at the middle of the nanotubes. The calculated ISS from this approach ranges from 106.7 to $142 \mathrm{MPa}$ for different CNT diameters. Sharma et al. [35] investigated the effect of the number of CNT walls on the interfacial properties of CNT-reinforced epoxy systems using MD. They found that the calculated ISS is greater in double-walled CNT when compared with single-walled CNT and triple-walled CNT. This is caused by the corresponding energy increment which is greater when the number of walls increases from one to two when compared with the energy increment from two to three. The reason can by justified by the increased distance between the newly inserted inner wall and the interface. Since the vdW interactions are dependent on the distance from the interface, the longer distance leads to weaker vdW interaction. Xiong and Meguid [36] investigated the interfacial mechanical characteristics of 
CNT-reinforced epoxy composites using MD simulations. They found that the ISS varies from 20 to $80 \mathrm{MPa}$ as they studied various parameters, such as epoxy density, length and diameter of the CNT, the CNT-epoxy interfacial thickness, Lennard-Jones (LJ) cut-off distance and capping conditions of a CNT. They found that The ISS of a nanocomposite decreases with the increase in the CNT length or diameter. This finding demonstrates the advantage of using smaller length and diameter of CNTs in nanocomposites. They also concluded that the CNT caps do not greatly influence pull-out forces unless fully surrounded by the matrix such that the additional atoms can participate in these interactions. Furthermore, the capped CNT has marginal influence on the vdW interaction energy while the polymeric end-cap affects such energy. Alian et al. [37] used a multiscale modeling scheme to study the effect of embedded CNT length, diameter, interfacial thickness, and LJ cut-off distance. Their reported ISS values are also found to be almost identical to those predicted by Wernik et al. [38] using atomistic-based continuum multiscale modeling technique.

\subsection{Buckling Behavior}

As mentioned in Section 2.1, considerable experimental and theoretical studies have been carried out to characterize the ISS of CNT-reinforced nanocomposites. However, significant discrepancies exist between experimental and theoretical results. These theoretically obtained values are remarkably different from the experimental data. Researchers attributed the discrepancy of the results to various anomalies in CNT-reinforced nanocomposites. The main anomalies include defects in CNTs such as vacancies and SW defect as well as buckled CNTs in nanocomposites inevitably introduced during CNT synthesis as well as manufacturing [39-41].

In particular, the diameters of CNTs are in the range of less than a nanometer for SWCNTs to about $30 \mathrm{~nm}$ for MWCNTs while the typical length of CNTs is on the order of microns [24, 42], leading to a large aspect ratio (length to diameter). Because of this large aspect ratio and hollow cylindrical structure, CNTs are prone to buckle under mechanical loads. Buckling can lead to failure in the form of a sudden decline in compressive load carrying capacity and undesirably distorted configuration of structures. Under axial compression, a CNT exhibits shell-like or beam-like buckling depending on its aspect ratio; shell-like buckling appears when the aspect ratio is relatively small, while beam-like buckling appears when the aspect ratio is relatively large. Numerous experimental and theoretical studies have investigated the buckling of CNTreinforced nanocomposites. Experimental researchers have observed graphs of CNTs in buckled 
state both as individual nanotubes [43-45] and when embedded in nanocomposites [46, 47]. A large volume of theoretical work, based on both continuum-based shell and beam theories [4850], and atomistic techniques such as MD [51-54], concentrates on buckling of nanotubes by themselves. However, an increasing number of researchers are interested in the compressive behavior of nanotubes when embedded in composites. For instance, Lourie et al. [46] coupled the classical Euler model with energy method to examine a CNT buckling behavior within a polymeric matrix. To account for the effect of transverse shear deformation during the CNT buckling, Zhang et al. [55] developed Timoshenko beam model, which offers a more precise prediction of the critical buckling stress. Ru [56] developed an elastic double-walled shell model for the buckling analysis of CNTs in an elastic matrix based on the classical shell theory [57]. Later, Liew and coworkers [58, 59] introduced a Pasternak foundation into Ru's model [56]. They proposed multi-walled shell model to describe the buckling behavior of MWCNTs that are embedded in a matrix with consideration of the van der Waals (vdW) interactions.

Since CNTs are highly prone to structural defects and buckling, many investigations have focused on buckling of defective CNTs. For example, The MD simulations were employed by Chandra and Namilae [60] to explore the compressive behavior of SWCNTs in the presence of chemical functionalization and SW defects. They found that functionalization and topological defects have a negative impact on the buckling stress of CNTs. The compressive behavior of SWCNTs and double-walled CNTs in the presence of vacancy defects was examined by Hao and coworkers $[61,62]$ using MD simulations. They found that the extent to which vacancy defects weaken the compressive load carrying capacity of CNTs is dependent on the length, chirality, and temperature of the nanotubes as well as the density of the defects and their relative position. Zhang et al. [63] conducted an investigation into the buckling behavior of SWCNTs with defects via MD simulations. They revealed that point defects cause a higher reduction in the buckling load than SW defects. Kulathunga et al. [64] examined the effects of various configurations of vacancy defects on the buckling of SWCNTs by MD simulations in different thermal environments. Their findings revealed that increasing number of missing atoms, asymmetry of vacancy configurations, and asymmetric distribution of vacancy clusters lead to higher deterioration in buckling behavior. Ranjbartoreh and Wang [65] studied the effects of length, radius, chirality, SW defect, and single vacancy defect on the buckling behavior of SWCNTs. Obtained results indicated that the axial stability of SWCNTs decreases significantly due to 
topological defects and the critical buckling strain was more susceptible to defects than the critical buckling force. Different aspect ratios of defective CNTs on the buckling behavior were investigated by Parvaneh et al. [66], who showed that single vacancy defects only have a weak impact on the critical buckling load of CNTs with a large aspect ratio at room temperature. Poelma et al. [67] combined analytical continuum theory with MD to study the effects of vacancy defect position on the CNT critical buckling load. They concluded that the defects at the ends of the CNT and close to the middle of the CNT significantly reduce the critical buckling load and strain at $1 \mathrm{~K}$. The compressive mechanical properties of SWCNTs with up to 20 randomly distributed vacancies were studied by Cheng et al. [68] using MD method. It was found that the Young's modulus of the SWCNTs is approximately linearly proportional to the number of vacancies and SWCNTs containing more vacancies have more complicated deformation procedures.

\subsection{The Need for the Current Studies \\ 2.3.1 Pull-out Investigation}

Evidently, many experimental, analytical, and numerical studies have been carried out to investigate the ISS of CNT-reinforced composites. However, significant discrepancies exist within the results, which may be due to the complexity of the problem and also to the oversimplifications in the numerical models. The CNT-polymer composite models are simplified for several reasons. Matrix materials are usually frozen during the pull-out process to avoid excessive degrees of freedom, but this leads to an inaccurate estimation of the interfacial thickness and the CNT-matrix interaction energy. Also, experimentally observed defects in CNTs are ignored; these include vacancies [16], SW [69] and interstitial atoms [70], which are inevitably introduced during CNT synthesis as well as manufacturing [39-41]. Also, covalent bonding between CNTs and matrix materials is not taken into consideration. Covalent bonding is usually introduced through surface functionalizations of CNTs [71-73], which are used to improve the load transfer ability of nanocomposites.

Another challenge is that most existing numerical models focus on thermoplastic polymers [8, 13, 15, 74]. There is an urgent need to develop numerical models which can accurately characterize the interfacial properties of CNT-reinforced thermoset composites. Thermoset materials generally have very different mechanical properties than thermoplastic materials because they comprise three-dimensional networks of bonds (cross-linking). However, to the 
best of authors' knowledge, there have been no studies performed on the effect of cross-link density on the interfacial properties of CNT-reinforced thermoset composites. Because these composites are important in many engineering applications, their neglect in the literature has motivated the current study.

The specific objective of this pull-out study is to develop MD simulations to investigate the effect of defects such as vacancies, carbon adatoms, and SW and of CNT functionalization on the ISS of CNT-reinforced epoxy polymers with different degrees of conversion. Molecular dynamics pull-out simulations were conducted at room temperature $(300 \mathrm{~K})$ to obtain the ISS for the above cases. During the pull-out process, the majority of the epoxy matrix surrounding the CNT was allowed to deform freely to capture the effect of matrix deformation on the interfacial properties of the nanocomposites investigated.

\subsubsection{Compressive Load Investigation}

It is evident from the foregoing literature review that there has been no work on the effect of various defects on the buckling of embedded CNTs. To the authors' knowledge, only a few studies have used MD simulations to focus on the buckling of embedded pristine CNTs. For instance, Namilae and Chandra [75] studied the compressive behavior of CNTs when they are embedded in PE matrix and with interface chemical modifications using MD simulations. It is observed that the buckling load for buckling increases only very marginally for nanotubes embedded in PE matrix compared with neat CNTs. In the case of chemically bonded interfaces, the critical stress for buckling is reduced compared with neat CNTs because of the changes in curvature introduced by chemical bonding. The buckling of SWCNTs embedded in PE matrix was examined by Kulathunga and Ang [76] via MD method. They found that the embedded CNT has higher buckling stress compared to the freestanding CNT. This stress increment is greater in CNTs with smaller aspect ratio. It is clear that there exists a necessity to carry out a comprehensive research on the buckling of defective CNTs embedded in polymer nanocomposites. The importance of CNT-reinforced nanocomposites to many engineering applications as well as the lack of coverage in the literature has motivated the current study.

The specific aim of this compressive load study is to examine the effect of defects such as vacancy and SW defect on the buckling behavior of CNTs embedded in an epoxy matrix. Defects with different densities and configurations were modeled. The MD simulations were 
performed at room temperature $(300 \mathrm{~K})$ based on the consistent valence forcefield (CVFF). To address the chirality effect, we considered zigzag and armchair SWCNTs with similar diameters. During the compressive loading process, the majority of the epoxy matrix surrounding the CNT was allowed to deform freely to capture the effect of matrix deformation on the buckling of the embedded CNTs accurately. 


\section{Chapter 3 \\ Fundamental Aspects of MD Simulation Techniques}

Summary: In this chapter, we describe the main features of molecular modeling of pull-out and compressive load simulations in details. Specifically, we describe the development of various types of defects in CNTs and modeling of the epoxy. Furthermore, we provide the steps adopted for the development and construction of the RVE.

\subsection{Numerical Simulation Techniques}

CNT pull-out test has been recognized as a standard method for evaluating the ISS of nanocomposites. The force or energy required to de-bond the CNTs from the surrounding matrix is the output of these tests which are used to calculate the ISS. Due to the difficulties associated with setting up nanoscale experiments, many researchers resort to atomistic methods such as MM and MD simulations to investigate the influence of defects and functionalization on the ISS of nanocomposites. In MD and MM techniques, the atomic structures of nanocomposites are initially defined. Interactions of atoms in nanocomposites are defined by interatomic potential energy function which is either obtained from first-principle calculations [77], or from experimental results, such as bond energy and atomization energy [78]. The interatomic potential energy is a function of the locations of nanocomposite atoms, and the gradient of the total potential is equal to the force on each atom. In MM, thermodynamic equilibrium is realized by minimizing the total potential energy of the system by modifying the atomic coordinates. The governing equation for $\mathrm{MM}$ is given by

$$
\frac{\partial U(\boldsymbol{r})}{\partial \boldsymbol{r}}=0
$$

where $U$ is the total potential energy of the system and $\boldsymbol{r}$ is the position vector of an atom. MM does not take into consideration time-dependent quantities like velocity, acceleration, and inertia force. In contrast to MM, MD is performed by solving Newton's equation of motion for each atom in the system. The governing equations for MD are provided by

$$
m_{i} \frac{d^{2} \boldsymbol{r}_{i}}{d t^{2}}=\boldsymbol{F}_{i}
$$

and 


$$
\boldsymbol{F}_{i}=-\frac{\partial U\left(\boldsymbol{r}_{1}, \boldsymbol{r}_{2}, \ldots, \boldsymbol{r}_{i}, \ldots, \boldsymbol{r}_{N}\right)}{\partial \boldsymbol{r}_{i}}
$$

where $\boldsymbol{r}_{i}$ is the position vector of atom $i, N$ is the total number of atoms, and $\boldsymbol{F}_{i}$ is the force vector acting on atom $i, m_{i}$ is the mass of ith atom, and $t$ is the time. Since MM does not consider transient and dynamic quantities, it is not able to account for the thermal effect during the CNT pull-out process. In order to determine the ISS accurately under realistic temperature, MD approach is performed by the authors in this research.

The current MD simulations were carried out using the large-scale atomic/molecular massively parallel simulator (LAMMPS) [79]. The CVFF [80] was adopted in the simulations to model all bonded and non-bonded interactions in the CNT-epoxy systems. This force field has been widely used by other researchers to study the mechanical properties of CNT-reinforced epoxy composites $[37,81]$. The analytic forms $\left(\mathrm{U}_{\mathrm{pot}}\right)$ of the energy expressions used in the CVFF are given below:

$$
U_{\text {pot }}=U_{\text {bond }}+U_{\text {angle }}+U_{\text {dihedral }}+U_{\text {improper }}+U_{v d W}+U_{\text {Coulomb }}
$$

where $U_{\text {bond }}, U_{\text {angle }}, U_{\text {dihedral }}, U_{\text {improper }}$ is the bonded interactions which represent the respective energy of deformation of bond lengths, bond angles, torsion angles and inversion angles, $U_{v d W}$ and $U_{\text {Coulomb }}$ is the non-bonded interactions which represent the respective $\mathrm{vdW}$ and electrostatic interactions. The components of the potential energy due to the bonded interactions are shown in Fig. 3.1.

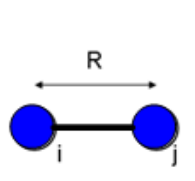

(a)

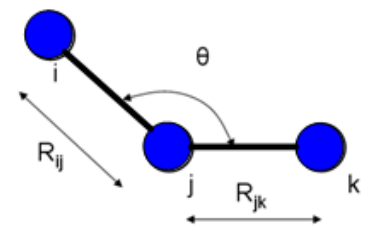

(b)

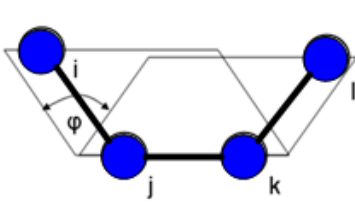

(c)

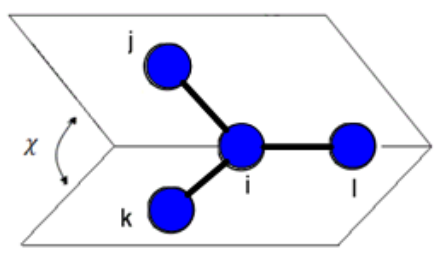

(d)

Fig. 3.1 Schematics of different bonded CVFF terms: (a) bond length, (b) bond angle, (c) torsion angle, (d) inversion angle.

\subsection{Molecular Modeling of Pull-out Simulation \\ 3.2.1 Molecular Structure of CNTs with Defects and Functionalization}

The molecular structures of five different types of possible defects and one type of CNT functionalization considered are presented in Fig. 3.2. All defects are assumed to form onto the 
sidewall of an armchair $(9,9)$ SWCNT. We studied SWCNTs because they are easy to handle and can reflect the effect of various defects and functionalization on the ISS better. The current CNT length is limited by the size of the RVE which is much smaller than its critical CNT length. It is also noted that the size of CNTs investigated is smaller than experimental studies due to the computational power limit; however, the current models can be extended to accommodate larger CNTs. As can be seen in Fig. 3.2(a)-3.2(c), monovacancy, bivacancy, and trivacancy were formed by removing one-, two- and three-bonded carbon atoms from the pristine SWCNT, respectively. A single adatom presented in Fig. 3.2(d) is an interstitial defect formed by an extra carbon that is covalently bonded to two adjacent carbon atoms. The SW defect shown in Fig. 3.2(e) is formed via a $90^{\circ}$ rotation of the carbon-carbon bond, transforming four hexagons into two pentagons and heptagons (5-7-7-5). Finally, Fig. 3.2(f) shows the phenyl functional group ($\mathrm{C}_{6} \mathrm{H}_{5}$ ) which is end-grafted to the sidewall of the CNT. The phenyl group contains a benzene ring, which typically exists in epoxy resins. The associated change in geometry of the atoms is that the phenyl group becomes perpendicular to the sidewalls of CNTs and the hybridization of the bonded atom is changed from $\mathrm{sp}^{2}$ to $\mathrm{sp}^{3}$.

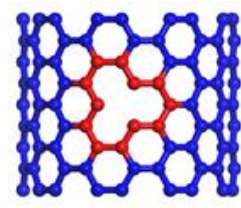

(a)

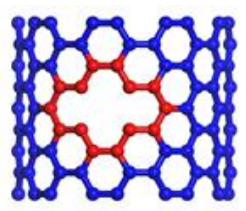

(b)

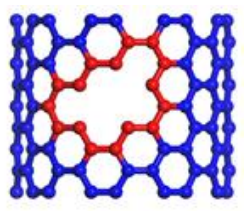

(c)

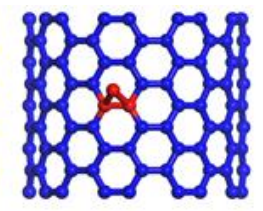

(d)

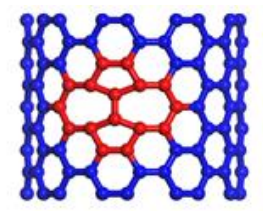

(e)

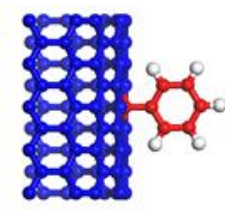

(f)

Fig. 3.2 Geometries of CNTs containing different defects and functionalization: (a) monovacancy, (b) bivacancy, (c) trivacancy, (d) single carbon adatom defect, (e) SW defect, and (f) phenyl group functionalization.

Five different defects of defective CNTs and one case of functionalization were considered as follows: (i) CNTs with 3, 6, 9, 12, 15, 18 monovacancies; (ii) CNTs with 2, 3, 4, 5, 6, 7, 8, 9 bivacancies; (iii) CNTs with 1, 2, 3, 4, 5, 6 trivacancies; (iv) CNTs with 4, 8, 12 adatoms; (v) CNTs with 5, 10, 15 SW defects; and (vi) Functionalized CNTs using 3, 6, 9 phenyl functional groups. The positions of the defects and functionalization were randomly located on the sidewalls of the CNTs. The simulation for each case was repeated three times, and the ISS results were averaged and curve fitted. 


\subsubsection{Cured versus Uncured Polymer}

The polymer used was a specific two-component epoxy resin based on a diglycidyl ether of bisphenol A (DGEBA) epoxy and triethylene tetramine (TETA) curing agent, which is typically used in the aerospace industry. Different degrees of conversion were introduced by analyzing both uncured and cured epoxy composites. Cured epoxy system was developed during the curing process by forming covalent bonds between the hydrogen atoms in the amine groups of the curing agent and the epoxide groups of the epoxy. As shown in Fig. 3.3, each epoxy oligomer consists of six DGEBA molecules connected by one TETA molecule so that the resin-hardener weight ratio in the epoxy polymer is equal to 100:16.7. Finally, 33 epoxy oligomers were packed into the RVE using Packmol software [82] to obtain an appropriate density, and the final structure of the RVE was visualized by VMD [83] as shown in Fig. 3.4(a). For the uncured epoxy matrix, the same numbers of individual DGEBA and TETA as the cured system were used to generate the RVE. The uncured state means that there is no curing process in the epoxy matrix and no covalent bonds were formed between the DGEBA and TETA.

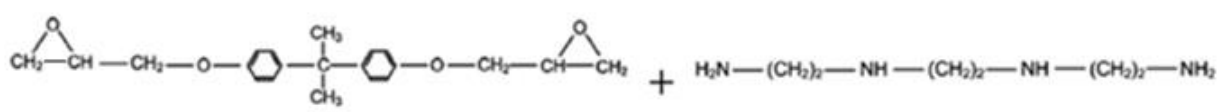

(a)

(b)

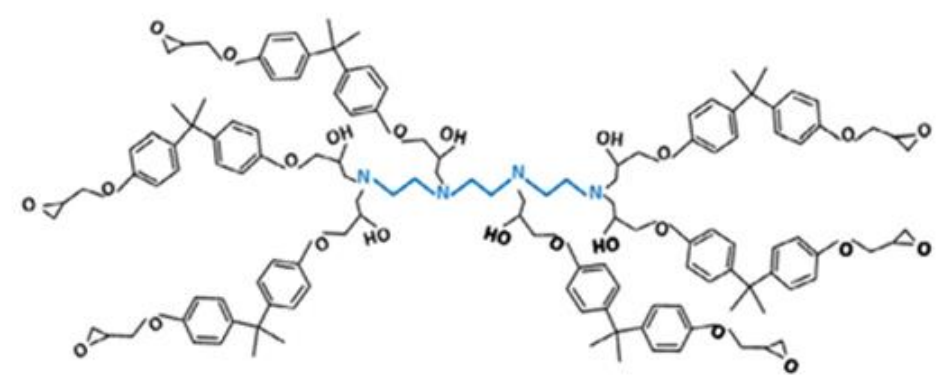

(c)

Fig. 3.3 Chemical structures of (a) epoxy resin (DGEBA), (b) curing agent (TETA), and (c) cured epoxy oligomer (6 DGEBA molecules connected by 1 TETA). 


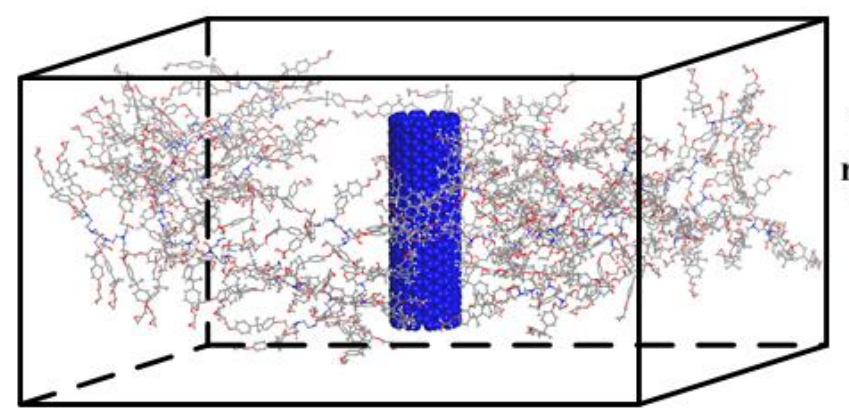

(a)

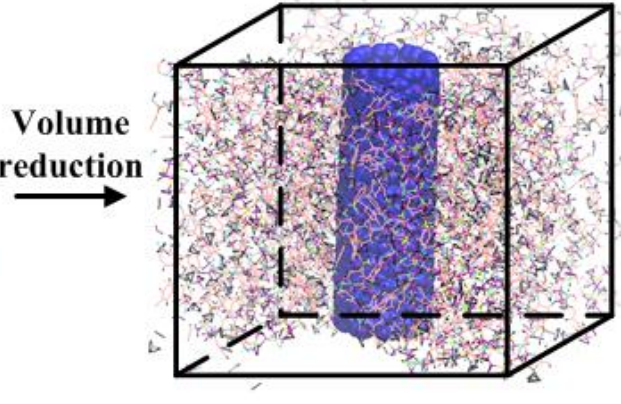

(b)

Fig. 3.4 Preparation of the RVE (a) SWCNT embedded in 33 randomly placed cured epoxy oligomers in a simulation box of size $150 \AA$ X $150 \AA$ X $45 \AA$, and (b) the compressed SWCNTepoxy system in a simulation box of size $50 \AA$ X $50 \AA$ X $42 \AA$.

\subsubsection{Construction of Nanocomposite RVE}

In order to conduct the pull-out simulations, a RVE was built to contain a SWCNT embedded at the center of the RVE and the epoxy polymer molecules. In all MD simulations, periodic boundary conditions were applied in the $\mathrm{x}$ - and $\mathrm{y}$-directions, while the z-direction was nonperiodic. The time step used in the MD simulations was selected to be $1 \mathrm{fs}$ for all MD simulations, and a cutoff distance of $12 \AA$ was used to model vdW interactions. The main steps involved in preparing the pull-out simulations for both uncured and cured epoxy composites are as follows:

Step 1 - Volume reduction: Since Packmol software treats molecules as rigid bodies, the size of the RVE was initially enlarged to accommodate all the epoxy molecules and the CNT atoms. Subsequently, the RVE was compressed gradually through seven steps from its initial dimensions of $150 \AA$ X $150 \AA$ X $45 \AA$ to the targeted size of $50 \AA$ X $50 \AA$ X $42 \AA$, resulting in the appropriate density of the RVE. At each stage, the CNT was "frozen", and the coordinates of the epoxy atoms were remapped to fit inside the compressed box. The potential energy of the compressed box was then minimized using the conjugate gradient method. The final compressed box is demonstrated in Fig. 3.4(b).

Step 2 - Equilibration: The compressed box was initially equilibrated for $2 \mathrm{~ns}$ in the isothermalisobaric (NPT) ensemble at $300 \mathrm{~K}$ and $1 \mathrm{~atm}$ using the Nose-Hoover algorithm to distribute the molecules evenly due to the volume reduction. The equilibrium was ensured by using the following temperature cycle protocol. Two stepwise NPT cycles of heating and cooling (from 
$400 \mathrm{~K}$ to $300 \mathrm{~K}$ by a step of $25 \mathrm{~K}$ ) were performed with each step occupying $10 \mathrm{ps}$. This was followed by a simulation in the NPT ensemble for $1 \mathrm{~ns}$ at $300 \mathrm{~K}$ and 1 atm to generate an epoxy system with an appropriate CNT-matrix interfacial thickness. The final equilibrated densities for the uncured and cured epoxy composites were found to be $0.99 \mathrm{~g} / \mathrm{cm}^{3}$ and $0.94 \mathrm{~g} / \mathrm{cm}^{3}$, respectively, which are slightly less than expected due to the non-periodic boundary in the $\mathrm{z}$ direction of the RVE.

\subsection{Molecular Modeling of Compressive Load Simulation 3.3.1 Molecular Structure of CNTs with Defects}

It is expected that defects have an influence on buckling behavior of CNTs, since buckling is very sensitive to geometric imperfections. However, it is crucial to understand the severity of the effect of different defects on the buckling behavior of CNTs. For CNTs with vacancy and SW defect, several factors can be presumed to influence buckling; such as, the number and location of defects, the number of vacancy clusters and the distribution of these vacancy clusters. In order to examine the contribution of these factors, the defect configurations given in Fig. 3.5 are investigated. Specifically, vacancies coded as (1A), (2B), and (3A) are symmetric monovacancy, bivacancy, and trivacancy, respectively. Vacancies coded as (2C) and (2B) are asymmetric bivacancy and trivacancy, respectively. Both vacancies coded as (1B) and (1C) contain two monovacancies. The monovacancies (1B) locate exactly opposite to each other while the monovacancies (1C) locate towards one side of the nanotubes. Symmetric and Asymmetric SW defects are coded as (1SW-A) and (1SW-B), respectively. Armchair (7, 7), (9, 9) and zigzag (12, 0) SWCNTs with the same aspect ratio of 6.5 were considered here. Two types of armchair SWCNTs were repeated to confirm the simulation results, and zigzag $(12,0)$ SWCNTs were chosen to have similar diameters to $\operatorname{armchair}(7,7)$ SWCNTs. All defects were assumed to form onto the sidewall of SWCNTs. The defects were introduced approximately at the middle of the nanotubes since defects at the mid-length are proven to reduce buckling strain and buckling load to the greatest $[66,84]$. 


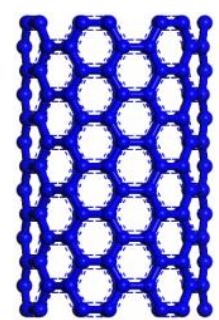

$(0 \mathrm{~A})$

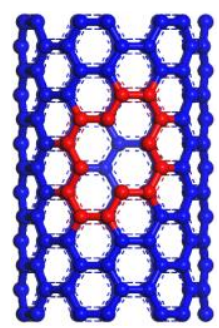

$(2 \mathrm{C})$

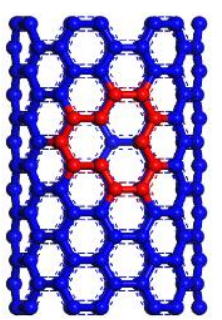

(1A)

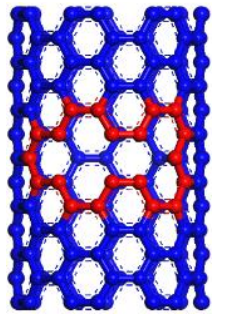

$(3 \mathrm{~A})$

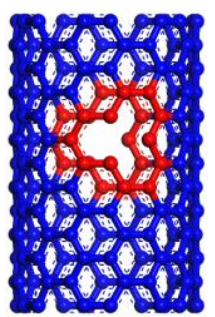

(1B)

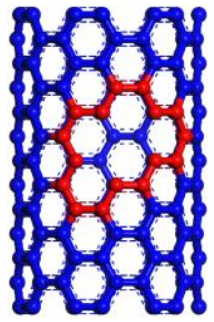

(3B)

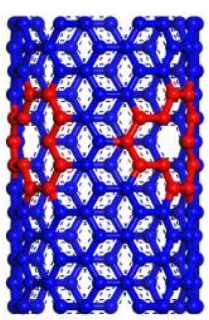

(1C)

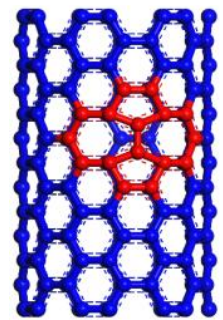

$(1 \mathrm{SW}-\mathrm{A})$

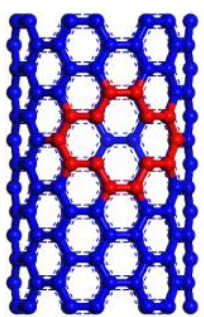

(2B)

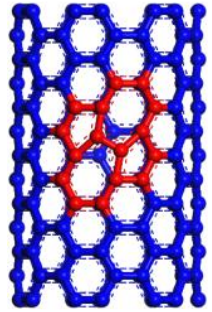

(1SW-B)

Fig. 3.5 Defects studied in this research $(0 \mathrm{~A}$ - pristine, $1 \mathrm{~A}$ - monovacancy, 1B - symmetrically distributed two monovacancies, 1C - asymmetrically distributed two monovacancies, $2 \mathrm{~B}$ symmetric bivacancy, 2C - asymmetric bivacancy, 3A - symmetric trivacancy, 3B - asymmetric trivacancy, 1SW-A - symmetric SW defect, 1SW-B - asymmetric SW defect).

\subsubsection{Construction of Freestanding CNT and Nanocomposite RVE}

It should be emphasized that the objective of the present study is to investigate the buckling behavior of an embedded CNT within a matrix and not the buckling behavior of the CNTreinforced nanocomposites. Thus, both freestanding CNTs and CNT-epoxy nanocomposites were prepared here with the view to understand the influence of matrix material on the buckling of defective CNTs. To prepare freestanding armchair $(7,7),(9,9)$ and zigzag $(12,0)$ SWCNTs for compression as discussed in Section 3.3.1, the SWCNTs were initially minimized in terms of total potential energy using the conjugate gradient method. This was followed by canonical (NVT) equilibration at $300 \mathrm{~K}$ for the duration of $50 \mathrm{ps}$ using a time step of $1 \mathrm{fs}$. The boundary condition was kept non-periodic in all three directions, and vdW interactions were truncated at a cutoff distance of $12 \AA$.

For the buckling simulation of embedded CNTs, the epoxy system was the same as the one for the pull-out analysis. In this MD simulation, periodic boundary conditions were applied in $\mathrm{X}$ - and y-directions, while periodicity was removed in z-direction. The time step used in the MD simulation was selected to be $1 \mathrm{fs}$, and a cutoff distance of $12 \AA$ was used to model vdW 
interactions. The same parameters were followed for different RVEs containing different armchair $(7,7),(9,9)$ and zigzag $(12,0)$ SWCNTs. For example, the main steps involved in preparing the buckling simulation for the $(7,7)$ pristine SWCNT-epoxy nanocomposites are as follows:

Step 1 - Volume reduction: Since Packmol software treats molecules as rigid bodies, the size of the RVE was initially enlarged to accommodate all the epoxy molecules and the CNT atoms. Subsequently, the RVE was compressed gradually through seven steps from its initial dimensions of $150 \AA$ X $150 \AA$ X $62 \AA$ to the targeted size of $50 \AA$ X $50 \AA$ X $62 \AA$, resulting in the appropriate density of the RVE. At each stage, the CNT was "frozen", and the coordinates of the epoxy atoms were remapped to fit inside the compressed box. The potential energy of the compressed box was then minimized using the conjugate gradient method.

Step 2 - Equilibration: The compressed box was initially equilibrated for $2 \mathrm{~ns}$ in the isothermalisobaric (NPT) ensemble at $300 \mathrm{~K}$ and 1 atm using the Nose-Hoover algorithm to distribute the molecules evenly due to the volume reduction. The equilibrium was ensured by using the following temperature cycle protocol. Two stepwise NPT cycles of heating and cooling (from $400 \mathrm{~K}$ to $300 \mathrm{~K}$ by a step of $25 \mathrm{~K}$ ) were performed with each step occupying $10 \mathrm{ps}$. This was followed by a simulation in the NPT ensemble for $1 \mathrm{~ns}$ at $300 \mathrm{~K}$ and $1 \mathrm{~atm}$ to generate an epoxy system with the appropriate CNT-matrix interfacial thickness. The final equilibrated density of the SWCNT-epoxy RVE was found to be $0.95 \mathrm{~g} / \mathrm{cm}^{3}$, which is slightly less than expected due to the non-periodic boundary in the z-direction of the RVE. 


\section{Chapter 4 \\ Molecular Dynamics Simulations}

Summary: In this chapter, we describe the details of the MD simulations adopted in conducting the numerical pull-out and compressive load numerical experiments. Furthermore, we discuss the outcome of these experiments concerning the effectiveness of CNT reinforcement on the ISS and the buckling behavior of CNT-reinforced composites.

\subsection{Pull-out Simulation 4.1.1 CNT Pull-out Method}

The MD pull-out numerical experiments were carried out to determine the ISS of both uncured and cured epoxy nanocomposite systems considered. To prevent the simultaneous drift of the epoxy with the CNT in the z-direction during the pull-out process, we constrained one edge of the epoxy molecules (shown in red in Fig. 4.1). In order to avoid the effect of the constraint on the final results, the constrained system was further equilibrated in the NVT ensemble at $300 \mathrm{~K}$ for $50 \mathrm{ps}$. The composition of uncured and cured RVEs prior to the pull-out process of the pristine CNT is listed in Table 1. Subsequently, the edge atoms of the CNT, which are marked in green in Fig. 4.1, were pulled out at a uniform velocity of $1 \mathrm{X}_{10^{-4}} \AA / \mathrm{fs}$ at $300 \mathrm{~K}$ in the NVT ensemble until the CNT was completely pulled out from the matrix. During the pull-out process, the driving forces formed by the CNT edge atoms pulled out the remaining CNT atoms in the zdirection. Since the interactive deformation of the CNT and the epoxy atoms is enabled, our method can capture the effect of each defect and functionalization accurately on the interfacial load transfer mechanisms in a deformable epoxy polymer matrix.

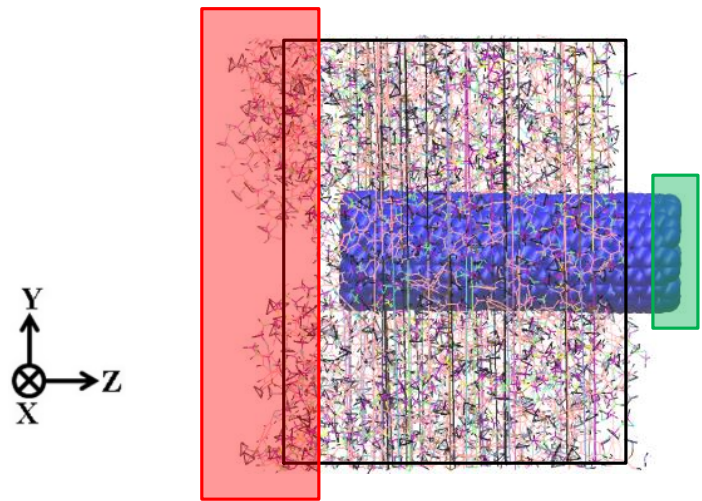

Fixed epoxy edge
Edge atoms for pull-out test

Fig. 4.1 Simulation setup for the pull-out of a CNT from the epoxy matrix. 
Table 4.1 Composition of uncured and cured nanocomposite RVEs immediately before pristine CNT pull-out simulation

\begin{tabular}{|c|c|c|c|c|c|c|c|c|}
\hline \multicolumn{3}{|l|}{ CNT } & \multirow{2}{*}{$\begin{array}{l}\text { No. of } \\
\text { chain }\end{array}$} & \multicolumn{5}{|c|}{ Composites } \\
\hline Chiral vector & Length $(\AA)$ & Radius $(\AA)$ & & $X(\AA)$ & $\mathrm{Y}(\AA)$ & $Z(\AA)$ & $\begin{array}{l}\text { Density } \\
\left(\mathrm{g} / \mathrm{cm}^{3}\right)\end{array}$ & $\begin{array}{l}\text { Total } \\
\text { number of } \\
\text { atoms }\end{array}$ \\
\hline$(9,9)$ & 38.176 & 5.751 & $\begin{array}{l}132 \text { (DGEBA) } \\
33 \text { (TETA) }\end{array}$ & 48.6 & 48.6 & 56.5 & 0.99 & 11238 \\
\hline$(9,9)$ & 38.120 & 5.752 & $\begin{array}{l}33 \text { (epoxy } \\
\text { oligomer) }\end{array}$ & 52.3 & 52.3 & 51.1 & 0.94 & 11238 \\
\hline
\end{tabular}

\subsubsection{Evaluation of ISS}

An energy approach $[8,10,31]$ has commonly been used to predict the ISS of CNT-reinforced composites. Since the work required to pull the CNT out is attributed to the shear force between the CNT and the matrix, the ISS can be calculated from the pull-out work $\left(W_{\text {pull-out }}\right)$ as follows:

$$
W_{\text {pull-out }}=\int_{0}^{L} 2 \pi r(L-z) \tau_{i} d z=\pi r l^{2} \tau_{i}
$$

where $l$ is the embedded initial length of the CNT into the matrix, $r$ is the radius of the $\mathrm{CNT}, \tau_{i}$ is the ISS, and $z$ is the pull-out distance from the initial position to the final position of the CNT. The pull-out work is usually defined as the variations in the potential energy of the entire system after and prior to the pull-out process as follows:

$$
W_{\text {pull-out }}=E_{\text {potential }}^{\text {final }}-E_{\text {potential }}^{\text {initial }}
$$

The potential energy of the nanocomposite can be described as follows:

$$
E_{\text {potential }}=E_{\text {cnt }}+E_{\text {epoxy }}+E_{\text {interaction }}
$$

where $E_{c n t}, E_{\text {epoxy }}$ and $E_{\text {interaction }}$ are the potential energy of the CNT and epoxy as well as the interaction energy between the CNT and epoxy matrix, respectively. Since most of the atoms in the CNTs are electrically neutral and there are no bonded interactions between the CNT and the matrix, the CNT-matrix interaction energy is mostly attributed to nonbonded vdW interactions. Several researchers $[31,32]$ also determined the interfacial binding energy $\gamma$ between a CNT and the matrix which serves as another reliable indicator of the effect of defects and functionalization 
on the nanocomposites. The interfacial binding energy $\gamma$ per unit area can be obtained from the interaction energy ( $\left.E_{\text {interaction }}\right)$ scaled by twice the instantaneous contact area,

$$
\gamma=\frac{E_{\text {interaction }}}{2 A}
$$

where $A$ is the instantaneous contact area at each pull-out step. In order to characterize atom distributions in the RVE, a dimensionless relative concentration of specific types of atoms can be determined in the radial direction of the CNT. This can be done to define the relative atom density of an atom present versus distance from the center of the CNT. The relative concentration of an atom is defined as the atom density in a given sub-volume divided by the density of the same atom present in the entire RVE $[85,86]$,

$$
C=\frac{N_{\text {sub }}}{V_{\text {sub }}} \cdot \frac{V_{t o t}}{N_{\text {tot }}}
$$

where $C$ is the relative concentration of the atom, $N_{s u b}$ is the number of atoms in the subvolume, $V_{\text {sub }}$ is the volume of the sub-volume, $N_{\text {tot }}$ is the total number of those atoms in the RVE, and $V_{\text {tot }}$ is the total volume of the RVE. Since the dimensional changes of CNTs containing defects and functional groups influence the ISS, the diameter and length of defective and functionalized CNTs after the equilibration of epoxy systems were used in all MD simulations.

\subsection{Compressive Load Simulation 4.2.1 CNT and RVE Compressive Load Method}

The MD compression simulations were carried out to determine the critical buckling strain and buckling load of both freestanding SWCNTs and SWCNT-epoxy nanocomposite systems. To prevent the simultaneous drift of the SWCNT and epoxy atoms in the z-direction during the compression process, we constrained one end of the freestanding SWCNT and the RVE (shown in red in Fig. 4.2). In order to avoid the effect of the constraint on the final results, the constrained systems were further equilibrated in the NVT ensemble at $300 \mathrm{~K}$ for $50 \mathrm{ps}$. The composition of the freestanding SWCNTs and RVEs containing different SWCNTs prior to the compression process is listed in Table 4.2. After the equilibration was complete, an incremental displacement of $0.01 \AA$ was applied on the other end of the freestanding SWCNTs and RVEs, which are marked in green in Fig. 4.2. After each increment, the systems were relaxed for a 
period to achieve a new equilibrium state while keeping the two ends constrained. The loading was repeated at $300 \mathrm{~K}$ in the NVT ensemble until the buckling occurred. Similar to the pull-out simulation, our method can capture the effect of each defect accurately on the buckling behavior of CNTs embedded in a deformable epoxy polymer matrix.

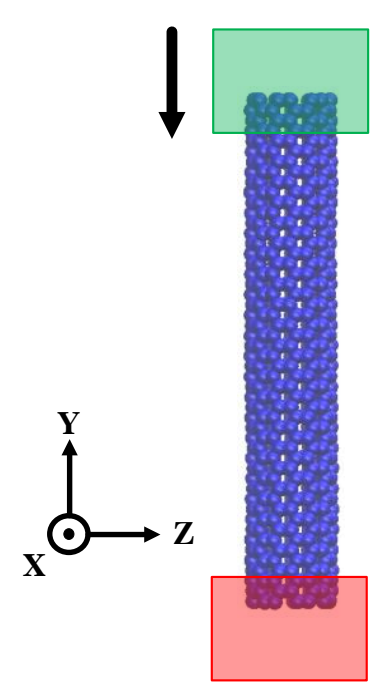

(a)

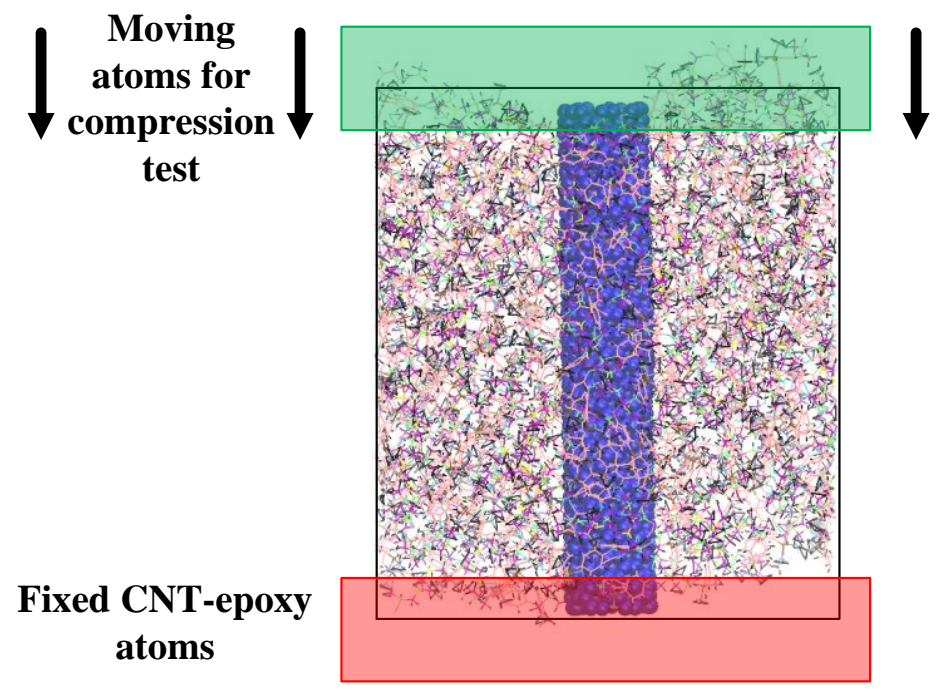

(b)

Fig. 4.2 Setup for the buckling simulations of (a) freestanding SWCNT, and (b) SWCNT embedded in the epoxy matrix.

Table 4.2 Composition of freestanding SWCNTs and RVEs containing different SWCNTs immediately before compression simulations.

\begin{tabular}{|c|c|c|c|c|c|c|c|c|}
\hline \multicolumn{3}{|c|}{ SWCNT } & \multirow{2}{*}{$\begin{array}{l}\text { Epoxy } \\
\text { No. of chain }\end{array}$} & \multicolumn{5}{|c|}{ Composites } \\
\hline $\begin{array}{l}\text { Chiral } \\
\text { vector }\end{array}$ & Length $(\AA)$ & Radius $(\AA)$ & & $X(\AA)$ & $Y(\AA)$ & $\mathrm{Z}(\AA)$ & $\begin{array}{l}\text { Density } \\
\left(\mathrm{g} / \mathrm{cm}^{3}\right)\end{array}$ & $\begin{array}{l}\text { Total } \\
\text { number of atoms }\end{array}$ \\
\hline$(7,7)$ & 55.89 & 4.46 & - & - & - & - & - & 700 \\
\hline$(9,9)$ & 72.11 & 5.73 & - & - & - & - & - & 1152 \\
\hline$(12,0)$ & 56.68 & 4.39 & - & - & - & - & - & 696 \\
\hline$(7,7)$ & 55.47 & 4.48 & 44 & 52.47 & 52.47 & 66.79 & 0.95 & 14868 \\
\hline$(9,9)$ & 71.70 & 5.75 & 57 & 52.21 & 52.21 & 87.05 & 0.97 & 19506 \\
\hline$(12,0)$ & 55.97 & 4.45 & 44 & 52.47 & 52.47 & 67.12 & 0.94 & 14864 \\
\hline
\end{tabular}

\subsubsection{Evaluation of Buckling Behavior}

As for the assessment of buckling behavior, the strain energy-strain and force-displacement curves for the pristine SWCNTs are shown in Fig. 4.3(a) and 4.3(b), respectively. The critical buckling state is detected by a sudden drop of strain energy or force. This sudden drop is 
associated with significant structural and geometrical changes of CNTs (shown in Fig. 4.4) corresponding to the release of energy in CNTs. It can be seen in Fig. 4.4 that different pristine SWCNTs with an aspect ratio of 6.5 buckle sideways with the occurrence of a flattening at the center, which is recognized in the literature as being "beam-shell buckling mode". It is a mixture of globalized beam-like buckling for a long and slender CNT and localized shell-like buckling within the atomic layer of a short CNT. In agreement with the findings of Wang et al. [54, 87], we conclude that CNTs with aspect ratios more than 6 exhibit beam-shell buckling mode, such as a single beam while preserving its circular cross section with localized shell buckling within the atomic layer. From Fig. 4.3(a), it is observed that the strain energy can be approximately expressed as a quadric function of the strain before the singularity happens in each curve, viz;

$$
U_{s}=\frac{1}{2} E A_{c} L \varepsilon^{2}
$$

where $U_{s}, E, A_{c}, L$, and $\varepsilon$ are the strain energy, Young's modulus, cross-sectional area, original length, and strain of the nanotubes, respectively. This indicates that the SWCNTs sustain elastic deformation under a compressive force in accordance with Hooke's law until bifurcation. Note that in Fig. 4.3(a) the critical buckling strain is defined as the strain at which the strain energy of the simulated SWCNT registers its first sudden drop in value. We can also see in Fig. 4.3(b) that the compressive force which the SWCNTs can withstand increases almost linearly with the axial displacement until the buckling load is reached. Accordingly, this relationship can be formulated as

$$
F=\frac{E A}{L} \Delta L
$$

where $F$ is the compressive force and $\Delta L$ is the end-shortening displacement. As it is shown in Fig. 4.3(b), there are two values of axial load $P$ at the critical buckling state. The upper value of $P$ at the critical buckling state is defined as the critical buckling load $P_{c r}$ and the corresponding end-shortening displacement is defined as the critical buckling displacement $\Delta_{\mathrm{cr}}$. Coincident with the value obtained through strain energy, the critical buckling strain $\varepsilon_{c r}$ can also be calculated by dividing $\Delta_{\mathrm{cr}}$ with the original length $L$ of CNT. 


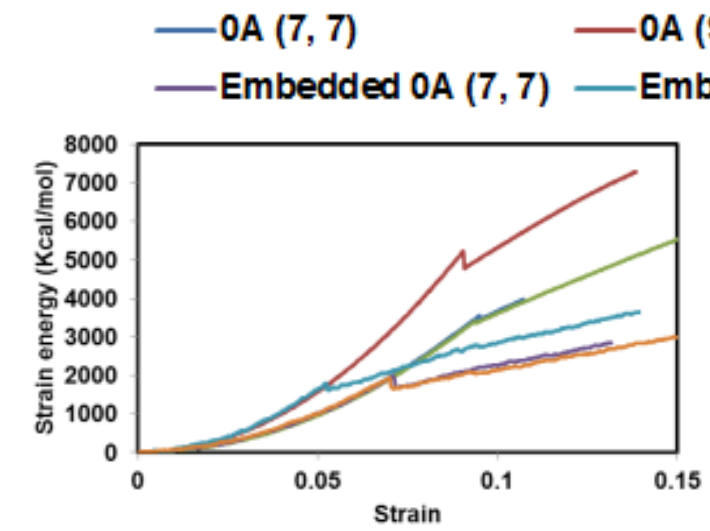

(a)

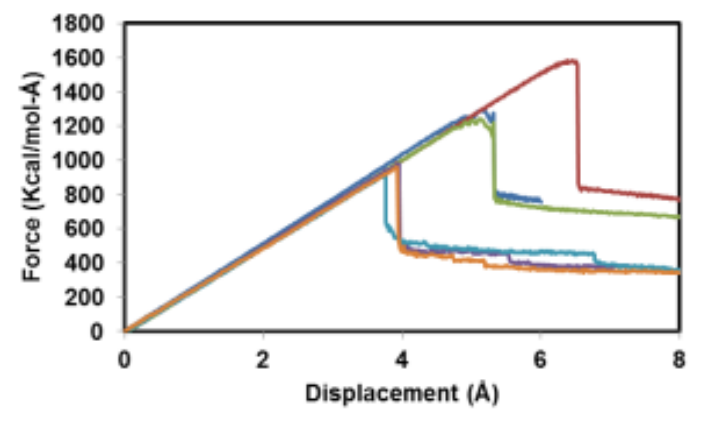

(b)

Fig. 4.3 (a) Strain energy-strain curves; (b) force-displacement curves for different pristine freestanding and embedded SWCNTs.
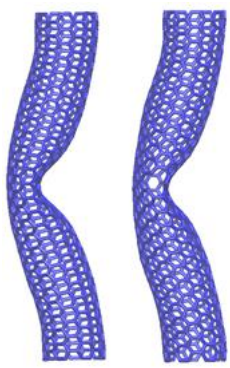

$\mathrm{OA}$
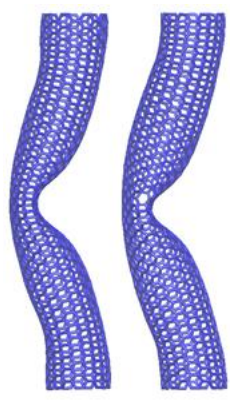

$1 \mathrm{~A}$

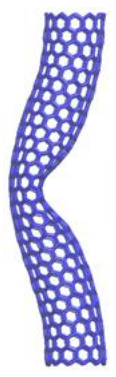

$\mathrm{OA}$

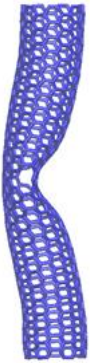

$1 \mathrm{~B}$

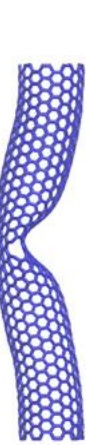

$1 \mathrm{~B}$

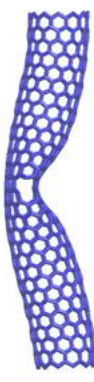

$1 \mathrm{~B}$
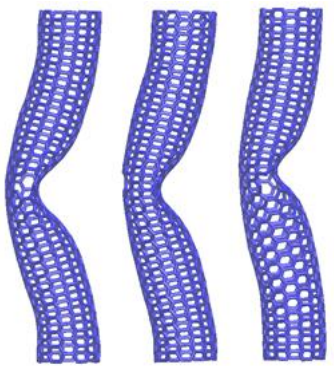

$2 \mathrm{C}$

(a) $(7,7)$
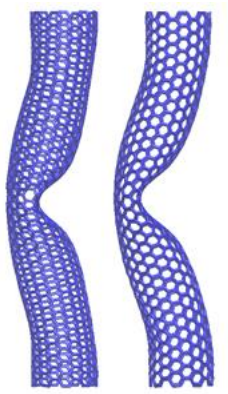

2B

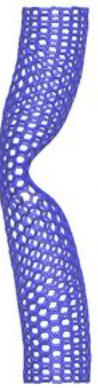

$2 \mathrm{C}$

(b) $(9,9)$
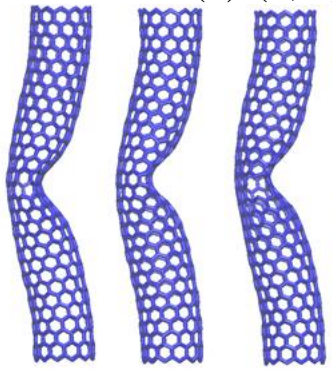

$2 \mathrm{~B} \quad 2 \mathrm{C}$

(c) $(12,0)$
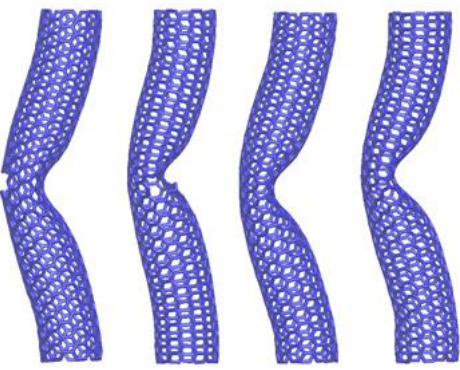

1SW-A 1SW-B
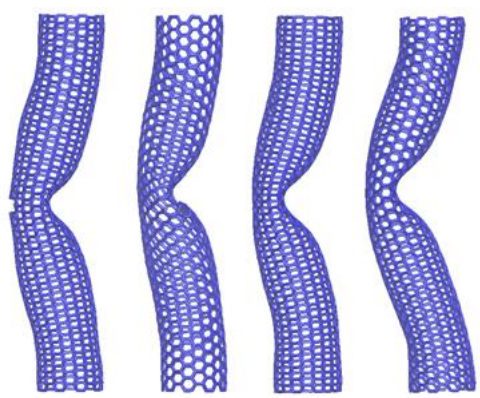

1SW-A 1SW-B
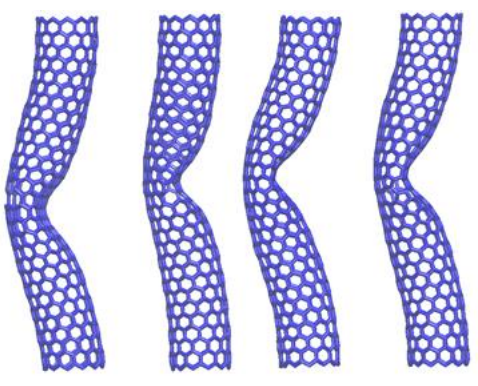

$3 \mathrm{~A}$

3B

1SW-A 1SW-B

Fig. 4.4 Buckling mode shapes of the freestanding SWCNTs (a) $(7,7)$, (b) $(9,9)$ and (c) $(12,0)$. 


\section{Chapter 5 \\ Results and Discussions}

Summary: In this chapter, we validate the proposed numerical simulations. Furthermore, we provide an insight into the results and identify the effects of such parameters as the vacancies, SW defects, carbon adatoms, and phenyl functional groups on CNT-reinforced composites.

\subsection{Analysis of Pull-out Simulation \\ 5.1.1 MD Model Validation}

Fig. 5.1 shows snapshots of the respective pull-out process of a pristine CNT from uncured and cured epoxy matrix. We can see that debonding takes place among the uncured epoxy molecules in close proximity to the CNT-matrix interface, and that a substantial number of uncured epoxy molecules are attached to the CNT. However, in the cured case, no epoxy molecules are pulled out along with the CNT. The respective change in the total potential energy of the uncured and cured CNT-epoxy systems is shown in Fig. 5.2. It can be observed that the potential energy of the cured system changes from approximately $-1000 \mathrm{Kcal} / \mathrm{mol}$ to zero at full pull out, while the corresponding uncured system changes from approximately $-700 \mathrm{Kcal} / \mathrm{mol}$ to zero again at full pull out of the CNT from the matrix. This is because the pull-out work is transferred into an increase in the potential energy of the system as the pull-out process proceeds. The reduced level of the change in potential energy of the uncured system can be explained by Fig. 5.1(a). The figure shows that some of the uncured molecules are attracted back to the RVE due to vdW forces. And, this happens before the CNT is pulled out completely from the matrix. 


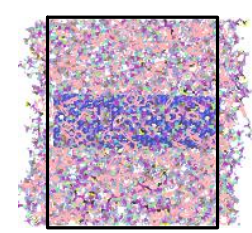

$\mathrm{z}=0$

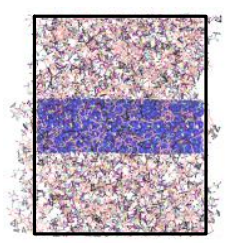

$\mathrm{z}=0$

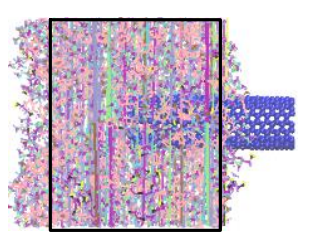

$\mathrm{Z}=20 \AA$

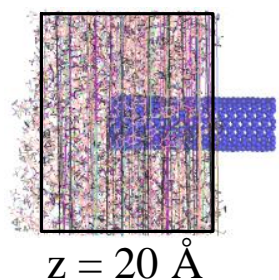

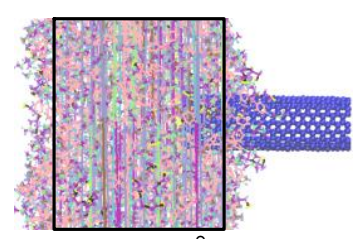

$\mathrm{Z}=30 \AA$

(a)

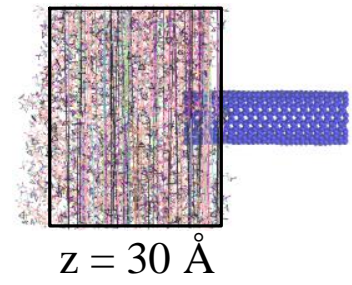

(b)
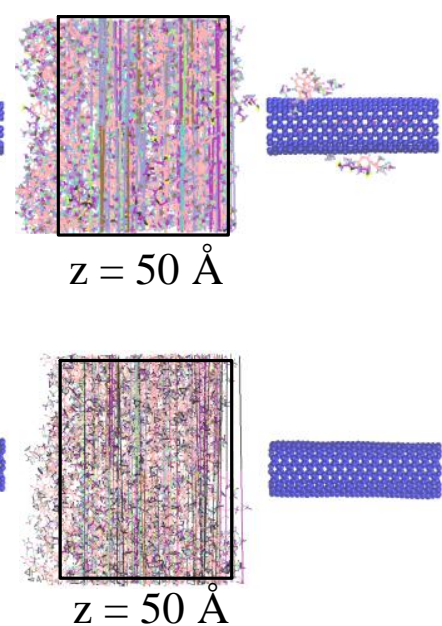

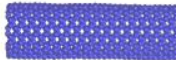

Fig. 5.1 Schematics of the pull-out processes of pristine CNT embedded in (a) uncured epoxy matrix and (b) cured epoxy matrix.

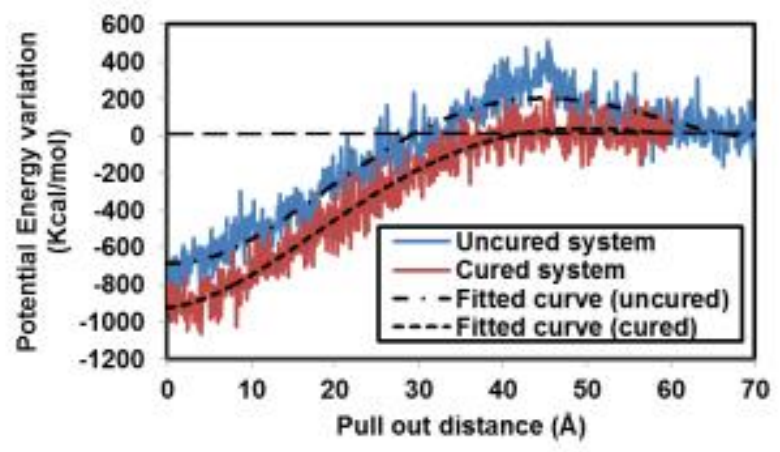

Fig. 5.2 Potential energy variations of pristine CNT with pull-out distance in the uncured and cured epoxy composites.

Based on the change of the total potential energy, the ISS of the uncured and cured epoxy composites was determined to be 198 and $250 \mathrm{MPa}$, respectively. The higher ISS of the cured system is because the epoxy resin is strengthened by the crosslinked structures developed during the curing process. The current ISS estimates for the pristine CNTs were found to be in good agreement with those of existing MD pull-out studies with some minor discrepancies due to the different forcefields used in the simulations. For instance, Bohlen and Bolton [88] used COMPASS forcefield to calculate the ISS via a new approach which assumed that the ISS was distributed uniformly within $10 \AA$ from each end of the SWCNT, but was zero at the middle of the nanotube. The ISS of a SWCNT-polyvinylidene fluoride system resulting from their 
approach was found to be $214.4 \mathrm{MPa}$ compared with $250 \mathrm{MPa}$ from the current study. Chowdhury and Okabe [89] used MD simulations to examine the effect of polymer matrix density on the ISS. The carbon-carbon interaction in the CNT was modeled using Brenner potential while AMBER potential was used for the polymer matrix. The ISS was calculated to be $245 \mathrm{MPa}$ for a SWCNT-PE composite with a density of $0.97 \mathrm{~g} / \mathrm{cm}^{3}$.

Fig. 5.3 shows the relative concentrations of all atoms and $\mathrm{sp}^{2}$ aromatic carbon atoms monitored as a function of the distance from the center of the CNT before and after crosslinking. As showed in Fig. 5.3(a), the relative concentration of all atoms for the uncured matrix near the CNT is higher than the cured one. The increase in the relative concentration is because a less crosslinked structure allows more conformational freedom for the matrix to interact favorably with the CNT. Enhanced conformational flexibility allows a more favorable and denser packing of matrix atoms with the CNT. Moreover, Fig. 5.3(b) reveals that it is actually the relative concentration of the $\mathrm{sp}^{2}$ carbon atoms that plays a major role in the CNT-matrix interaction energy. Such factors can improve the interaction energy at the CNT-epoxy interface. Fig. 5.4 compares the aromatic ring distribution near the CNT in the uncured and cured systems schematically. The red circles highlight typical aromatic ring conformation near the CNTs. We can see that aromatic rings in the uncured epoxy matrix are preferentially aligned parallel to the surface of the CNT, which optimizes $\pi$-stacking. The curing of the epoxy matrix sterically works against the aromatic rings lying flat on the surface of the CNT, reducing the interaction energy induced by $\pi-\pi$ attractions at the CNT-epoxy interface. However, higher CNT-epoxy interaction energy does not necessarily translate into higher ISS since the ISS also depends on the potential energy of the matrix and CNT in accordance with Eq. (4.4). This research examines the relative concentration of the $\mathrm{sp}^{2}$ carbon atoms to investigate the resulting interface properties better. 


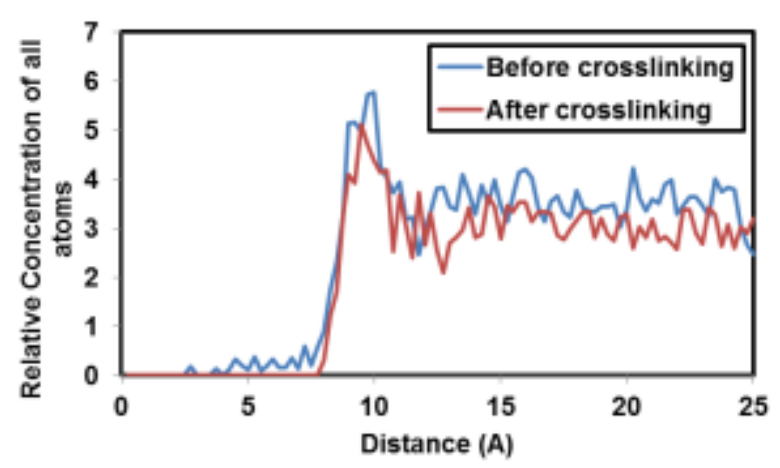

(a)

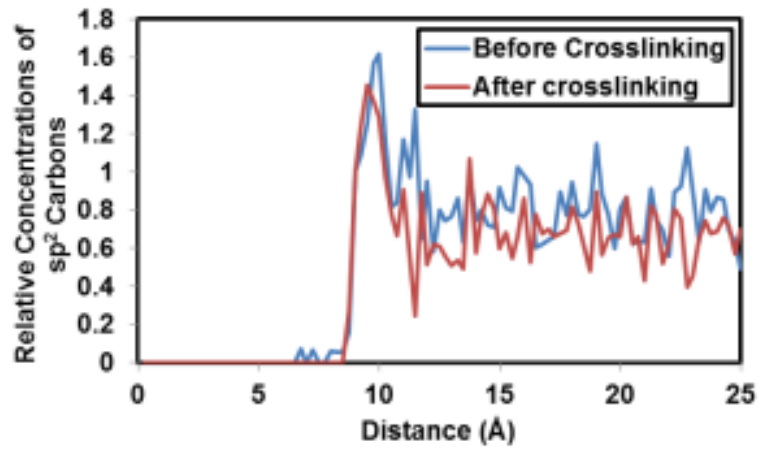

(b)

Fig. 5.3 Spatially averaged concentration profiles versus distance from the center of the CNT before and after curing (a) for all atoms and (b) for $\mathrm{sp}^{2}$-hybridized carbon atoms.
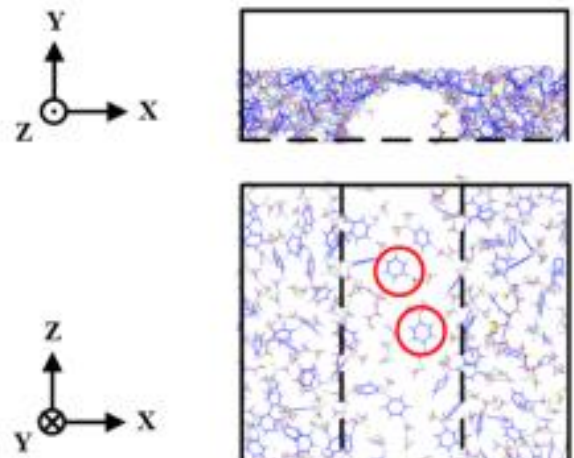

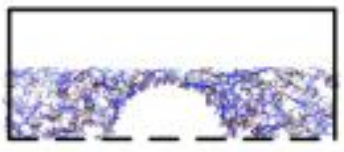

(a)

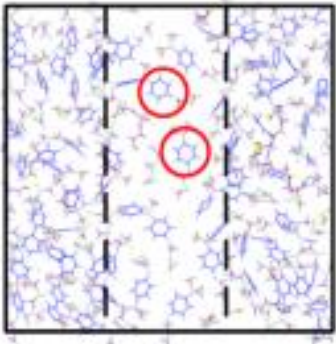

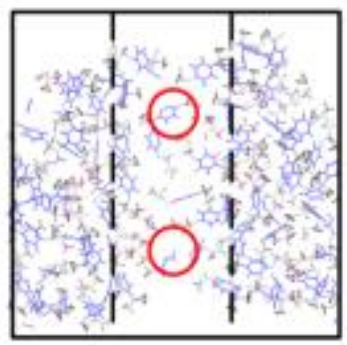

(b)

Fig. 5.4 Aromatic ring distribution near the CNT in (a) uncured epoxy matrix and (b) cured epoxy matrix.

\subsubsection{Effect of Vacancy Defects upon ISS}

Fig. 5.5 shows the variations in the ISS of an RVE containing a defective CNT with different numbers of missing atoms. It can be seen that the ISS of the cured systems decreases as the number of missing atoms in the CNTs increases irrespective of the type of defect. Since the potential energy of the epoxy and CNT does not alter very much for the cured systems, the interaction energy becomes the major contributor to the ISS. The vdW interaction between the CNT and the matrix solely contributes to the interfacial interaction energy. Thus, and as expected, the vdW interaction energy is reduced as a result of increasing number of vacancy defects in the CNT, which will eventually degrade the ISS. From Fig. 5.6(a-c), it is clear that the relative concentrations of $\mathrm{sp}^{2}$ carbon atoms near the CNT decreases with the increase in the number of vacancy defects, irrespective of the type of defect. An increasing number of vacancy 
defects decreases the number of $\mathrm{sp}^{2}$ carbon atoms in the CNT, which in turn reduces the ISS induced by $\pi-\pi$ attractions. Unlike the cured system, Fig. 5.5 shows that the number of vacancies in the uncured systems does not affect the ISS. This observation can be explained by the relatively low shear strength in the uncured systems. Similar to the uncured pristine CNT system, the debonding takes place initially among the epoxy molecules near the CNT-matrix interface during the pull-out process. Therefore, it is predominantly the uncured epoxy molecules rather than the CNT-epoxy interface that governs the shear strength of the interface.

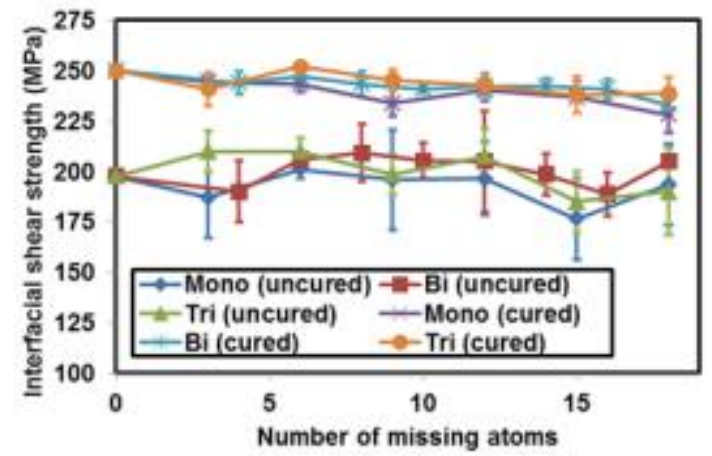

Fig. 5.5 Variations in ISS with number of missing atoms prior to and post curing.

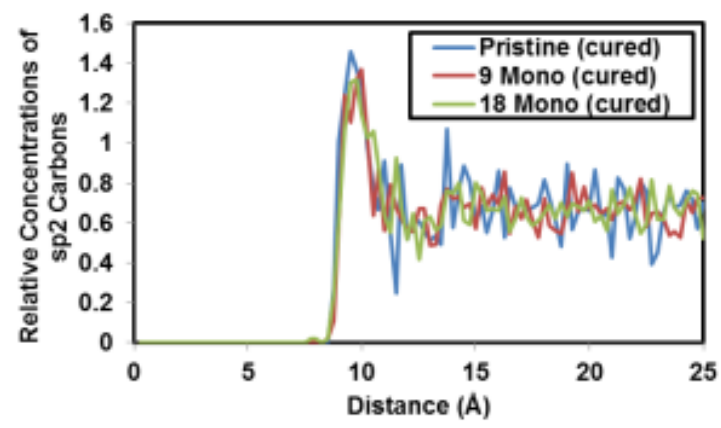

(a)

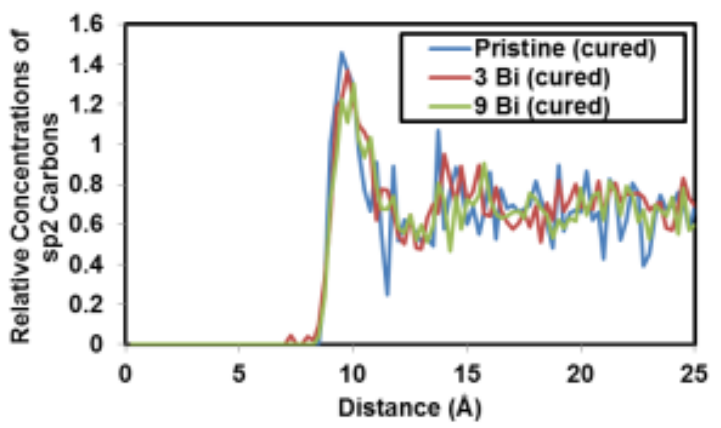

(b)

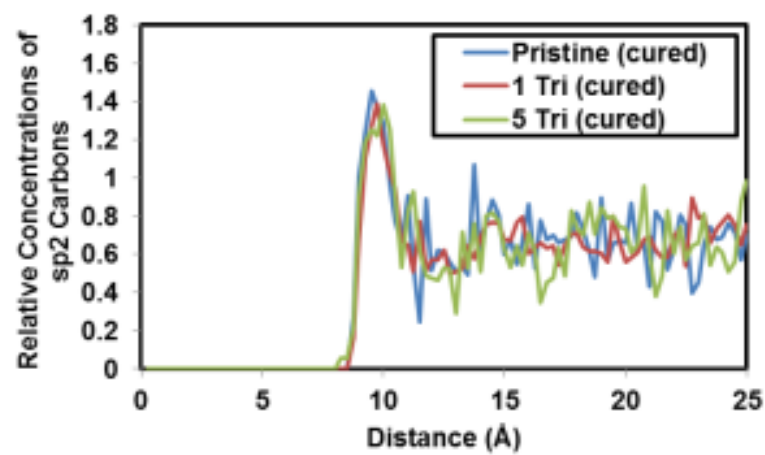

(c)

Fig. 5.6 Relative concentrations of $\mathrm{sp}^{2}$ carbon atoms for (a) different monovacancies after curing, (b) different bivacancies after curing, and (c) different trivacancies after curing. 
Our results in Fig. 5.5 also reveal that for the same number of missing atoms, different types of vacancy defects have almost the same effect on the ISS of the cured systems. We can see that the ISS for different types of vacancies decline at a similar rate and are overlapped for the most part of the curves. Therefore, it can be deduced that the decrease in the ISS is independent of the defect type. The fluctuation of the ISS is possibly due to the different distributions of vacancies within the CNTs and the surrounding epoxy molecules. A total of 18 missing atoms were considered in this study, and the average maximum percentage of decrease in the ISS of the cured epoxy matrix was found to be $7.0 \%$. Our findings generally agree with the earlier work of Yang et al. [10], but their work used COMPASS forcefield to study SWCNTs embedded in thermoplastic (PE) systems with no crosslinked structures. They reported $4.71 \%$ reduction of the ISS for 9 missing atoms. Chowdhury et al. [13] also used thermoplastic (PE) systems to examine three patterns of vacancy defects with different sizes and distributions; considering up to 8 missing atoms in SWCNTs. They found a maximum reduction in ISS of about $5.33 \%, 5.00 \%$ and $6.00 \%$ for one-atom, two-atom, and line vacancies, respectively.

Fig. 5.7 shows the interfacial binding energies of cured epoxy systems for the respective monovacancy, bivacancy and trivacancy defects in CNTs obtained from the CNT-matrix interaction energy divided by the instantaneous contact area, as defined in Eq. (4.5). In all cases, the magnitude of interfacial binding energy increases gradually as the CNT is pulled out from the matrix. The increase in the interfacial binding energy is due to the reduction in the contact area between the CNT and matrix during the pull-out process. These pulled out epoxy molecules further interact with the CNT, contributing to the increase in the interfacial binding energy. Furthermore, it can be observed that the magnitude of the interfacial binding energies in the case of vacancy defects in CNTs is initially smaller and then exceeds that of pristine CNTs as the pull-out distance for the CNT increases. The snapshots of the pull-out process presented in Fig. 5.8 show that some of the epoxy molecules entered the vacancy defective CNT through the uncapped ends leading to the enhancement of the interaction energy during the pull-out process. This results in a noticeable increase in the interfacial binding energy of vacancy defective CNTs during the pull-out process. 


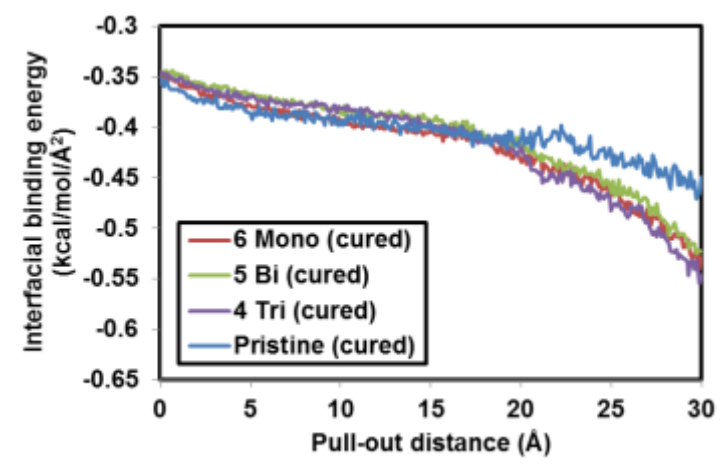

Fig. 5.7 Variations in interfacial binding energy between vacancy defective CNTs and cured epoxy matrix with pull-out distance.

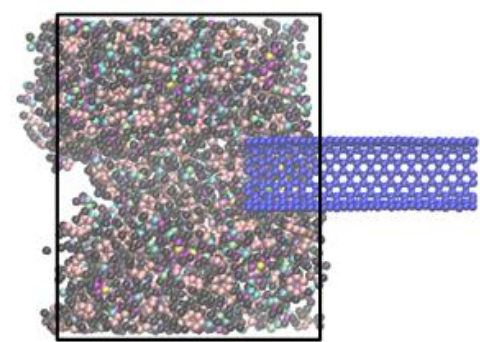

(a)

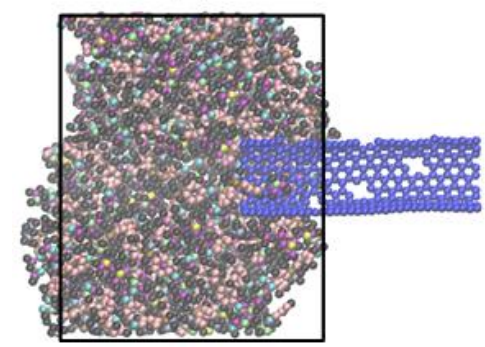

(c)

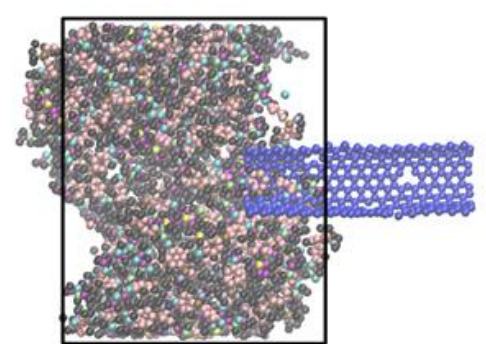

(b)

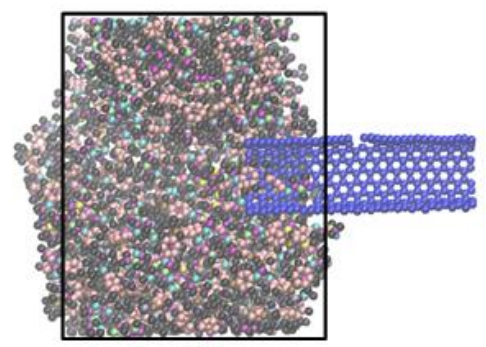

(d)

Fig. 5.8 Cross sections of the pull-out process for pristine and vacancy defective CNT in cured epoxy matrix at $\mathrm{z}=30 \AA$ : (a) pristine, (b) 6 monovacancies, (c) 5 bivacancies, and (d) 4 trivacancies.

\subsubsection{Effect of Carbon Adatoms upon ISS}

Unlike the case of vacancy defects, adatom defects have only a marginal influence on the ISS of the cured epoxy composites (see Fig. 5.9). This can be attributed to the rehybridization and the increase in the total number of carbon atoms in the adatom defective CNTs. Newly introduced carbon atoms rehybridize the adjacent carbon atoms and replace $2 \mathrm{sp}^{2}$ by $3 \mathrm{sp}^{3}$ carbon atoms. Although adatom defective CNTs have more carbon atoms, the unchanged ISS indicates that the interaction energy due to $3 \mathrm{sp}^{3}$ carbon atoms is similar to that due to $2 \mathrm{sp}^{2}$ carbon atoms. As 
shown in Fig. 5.10, the concentrations of $\mathrm{sp}^{2}$ carbons are almost the same for different numbers of adatoms in the cured matrix, an observation which helps to shed light on the marginal influence of adatom defects.

The results are generally consistent with those reported by Yang et al. [10], but their studies focused on thermoplastic (PE) systems without crosslinked structures. They found that adatom defects increase the ISS by only $1.1 \%$, and they also demonstrated that PE molecules are more effectively adsorbed on the sidewall of an adatom defective CNT than on that of a pristine CNT. Additionally, it is evident from Fig. 5.9 that adatom defective CNTs embedded in the uncured system have very similar ISS, which is analogous to the uncured case of vacancy defects.

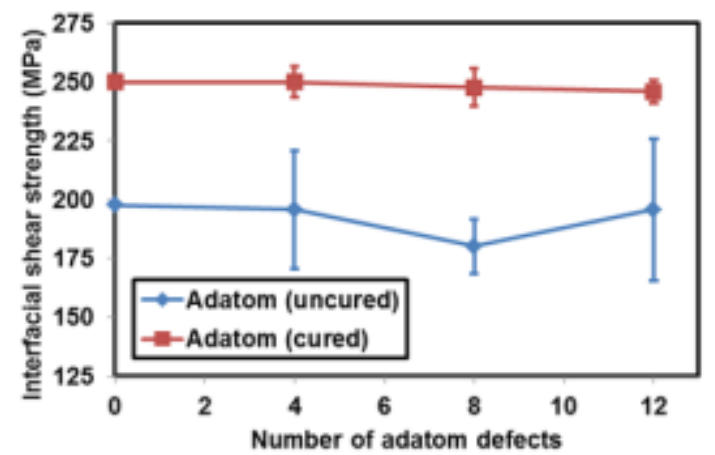

Fig. 5.9 Variations in ISS with number of adatom defects prior to and post curing.

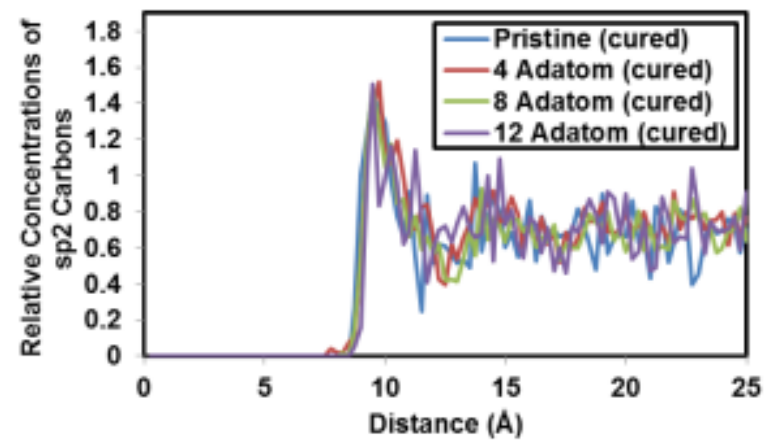

Fig. 5.10 Relative concentrations of $\mathrm{sp}^{2}$ carbon atoms for different adatoms after curing.

Fig. 5.11 shows the variations in the interfacial binding energies with the pull-out distance for the cured epoxy matrix. Analogous to the case of vacancy defects, the interfacial binding energies increase with the increase in the pull-out distance, and they further exceed that of pristine CNTs for larger pull-out distances. The noticeable increase in the interfacial binding energy can be explained by the fact that carbon adatoms on the sidewalls of the CNTs act like a 
spike, protruding in the surrounding epoxy molecules, which tends to pull out epoxy molecules of the RVE during the pull-out process in the cured epoxy matrix, as shown in Fig. 5.12.

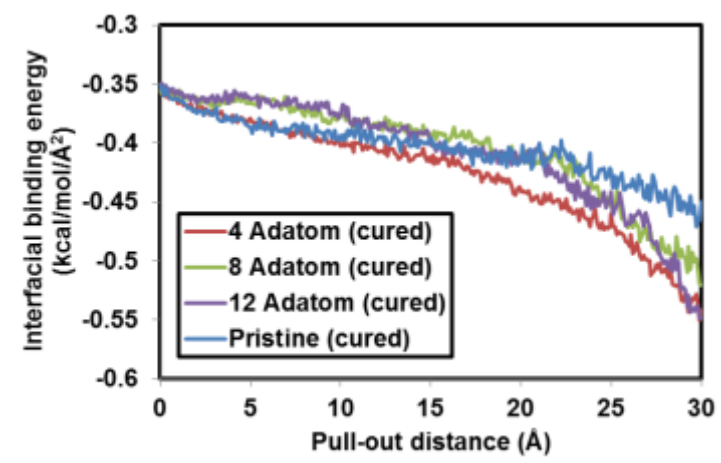

Fig. 5.11 Variations in interfacial binding energy between adatom defective CNTs and cured epoxy matrix with pull-out distance.

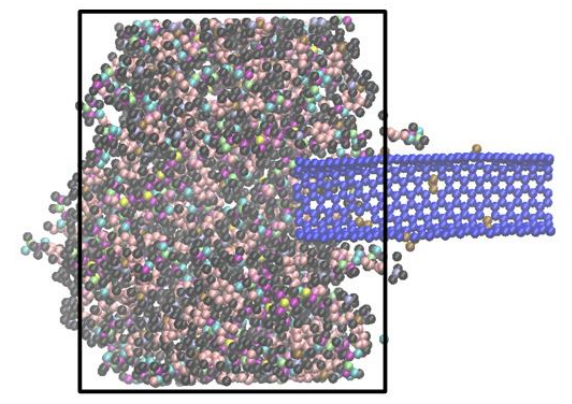

Fig. 5.12 Cross section of the pull-out process for 12 adatom defective CNT in cured epoxy matrix at $\mathrm{z}=30 \AA$.

\subsubsection{Effect of SW Defects upon ISS}

In contrast to the vacancy and adatom defects, the ISS of epoxy nanocomposite reinforced with CNTs containing SW defects increases as the number of SW defects increases for the cured epoxy systems (see Fig. 5.13). Note that the formation of SW defect involves neither missing carbon atoms nor the rehybridization of these atoms in the CNTs. In this case, four hexagons are transformed into two pentagons and two heptagons for a single SW defect. It may be observed from Fig. 5.13 that the maximum increase in the ISS of the cured systems is $6.0 \%$. Since the increase in the number of SW defects increases the concentrations of $\mathrm{sp}^{2}$ carbons near the CNT (Fig. 5.14), it can be concluded that the increase in the ISS results from the increase in $\pi-\pi$ attractions at the CNT-matrix interface. In addition, the ISS in the uncured matrix tends to be unvaried. This again is caused by the relatively low shear strength of the uncured epoxy systems. 


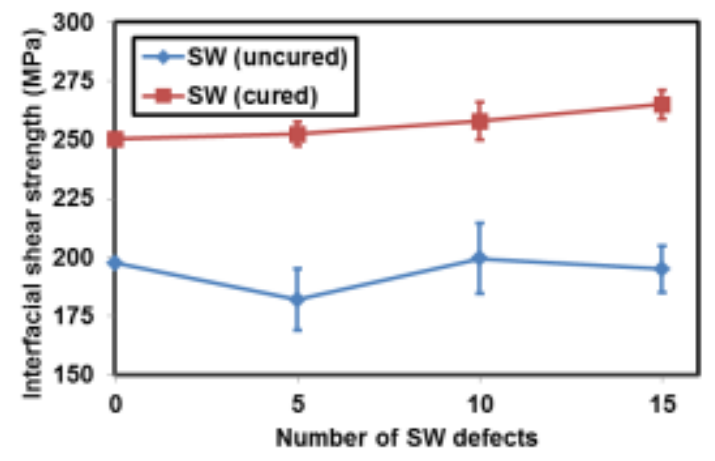

Fig. 5.13 Variations in ISS with number of SW defects prior to and post curing.

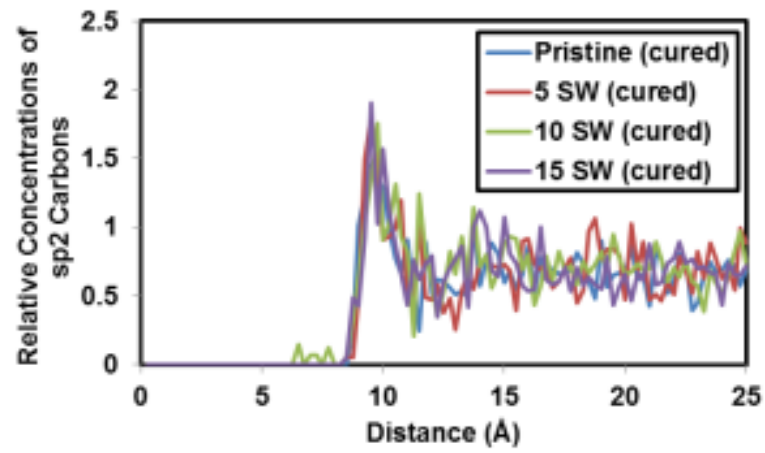

Fig. 5.14 Relative concentrations of $\mathrm{sp}^{2}$ carbon atoms for different SW defects after curing.

Our results are in agreement with the observation made by Yang et al. [10], though their work was concerned with thermoplastics (PE) with no crosslinked structures. They studied the effect of SW defects on the ISS of SWCNT-PE composite and found that the percentage increase in the ISS ranged from $0.7 \%$ to $7.1 \%$. Zhou and Shi [90] helped to explain the increase in the ISS by using a first-principle discrete variational method to estimate the binding energy of foreign atoms attached to CNTs with and without a SW defect. They found that SW defective CNT improves the binding energy magnitude by about $0.5 \mathrm{eV}$ for some foreign atoms compared with the pristine CNT. This would improve the adhesion of the polymer matrix onto the CNTs.

Fig. 5.15 shows the variations in the interfacial binding energies for the SW defective CNTs in the cured epoxy matrix against the CNT pull-out distance. Unlike the vacancy and adatom defective cases, the magnitude of the interfacial binding energies of this case is larger than that of the pristine CNTs, and the energy difference increases with the increase in the pull-out distance. This is attributed to the stronger adhesion effect of SW defective CNTs as mentioned above. Fig. 5.16 shows the cross section of the cured CNT-epoxy nanocomposite. We can see 
that some epoxy molecules adjacent to the sidewall of SW defective CNT are pulled out together with the CNT leading to an enhanced CNT-matrix interaction energy. This increase in the interaction energy leads to the increased interfacial binding energy difference between SW defective and pristine CNTs.

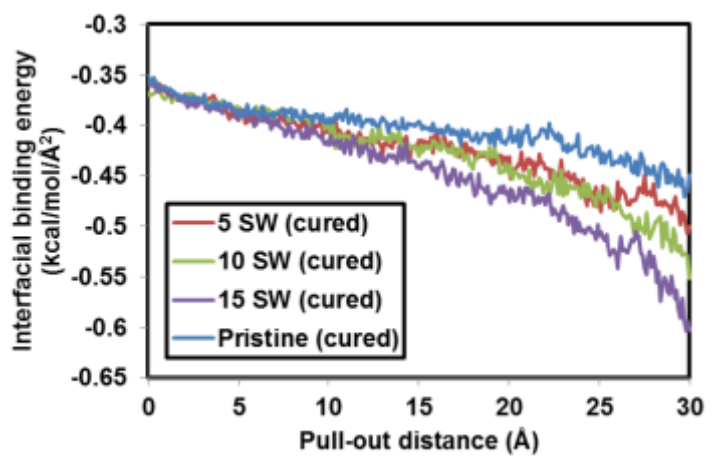

Fig. 5.15 Variations in interfacial binding energy between SW defective CNTs and cured epoxy matrix with pull-out distance.

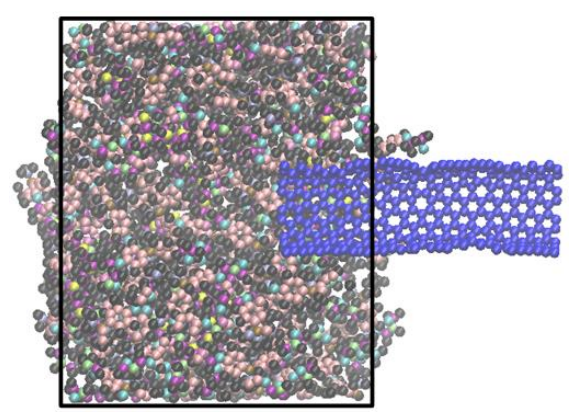

Fig. 5.16 Cross section of the pull-out process for $15 \mathrm{SW}$ defective CNT in cured epoxy matrix at $\mathrm{z}=30 \AA$.

\subsubsection{Effect of Phenyl Functional Groups upon ISS}

In this section, we establish the effect of functionalization on the ISS of CNT-epoxy nanocomposites. Fig. 5.17 shows that the ISS increases dramatically with the increase in the number of the phenyl group functionalization on the sidewalls of the CNTs for the cured epoxy composites. Noteworthy is the fact that the carbon atoms of the CNT are directly bonded to the phenyl group which changes the hybridization from $\mathrm{sp}^{2}$ to $\mathrm{sp}^{3}$, and this formation of the phenyl group functionalization adds more atoms to the original CNT. Moreover, Fig. 5.18 shows that the relative concentrations of $\mathrm{sp}^{2}$ carbons increase as the number of phenyl groups increases. This also results in the increase in the ISS induced by $\Omega-\Omega$ attractions. Therefore, the increased ISS is mainly attributed to the additional vdW interactions between the attached phenyl groups and the 
epoxy molecules. Unsurprisingly, phenyl functional groups do not improve the ISS of the uncured systems due to the same reasons mentioned above.

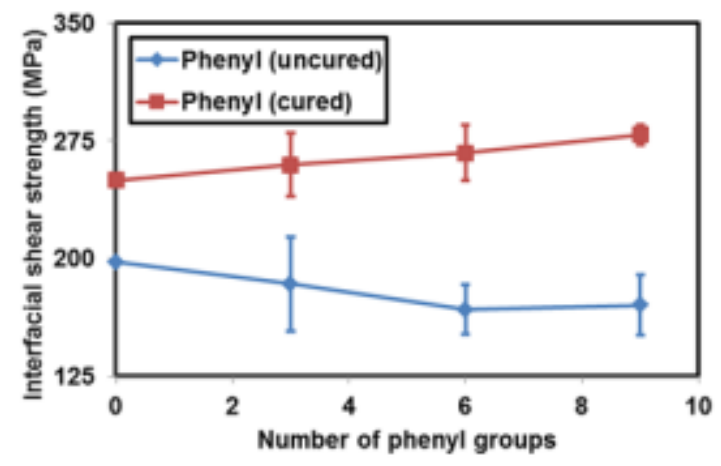

Fig. 5.17 Variations in ISS with number of phenyl groups prior to and post curing.

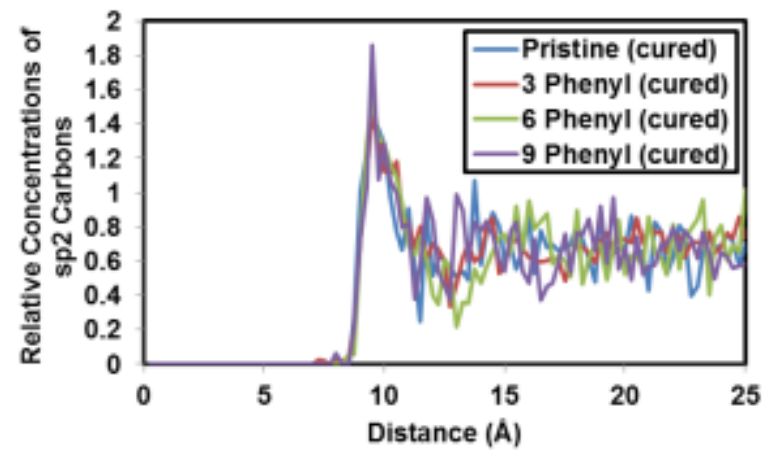

Fig. 5.18 Relative concentrations of $\mathrm{sp}^{2}$ carbon atoms for different phenyl groups after curing.

It can be observed from Fig. 5.17 that the ISS of the cured epoxy composites increases by $11.5 \%$ in comparison with the pristine CNT. Our results are found to be generally comparable with those reported in the literature despite different matrix materials and simulation techniques. However, most of them only paid attention to cured epoxies or thermoplastics (PE) with no crosslinked structures. For example, Sharma et al. [35] examined the effect of functionalization using Ethylene-di-amine $\left(\mathrm{E}-\mathrm{NH}_{2}\right)$ functional group on the interfacial bonding characteristics of CNT-epoxy composite. They found that the amine functionalized SWCNT increases the ISS twice the pristine SWCNT. Xiao et al. [74] studied the effects of different functionalization schemes on the interfacial strength of SWCNT-PE systems. They also found that the respective ISS is improved by $8.89 \%, 12.22 \%$, and $31.11 \%$ for three types of alkyl groups $\left(\mathrm{C}_{5} \mathrm{H}_{11} / \mathrm{C}_{10} \mathrm{H}_{21} / \mathrm{C}_{15} \mathrm{H}_{31}\right)$. Zheng et al. [8] used pull-out simulations to investigate the interfacial bonding characteristics between SWCNTs, on which $-\mathrm{COOH},-\mathrm{CONH}_{2},-\mathrm{C}_{6} \mathrm{H}_{11}$, or $-\mathrm{C}_{6} \mathrm{H}_{5}$ groups were chemically attached, and the PE matrix. They found that the ISS for $-\mathrm{C}_{6} \mathrm{H}_{11}$ and - 
$\mathrm{C}_{6} \mathrm{H}_{5}$ functionalized groups increases 3 times and 17 times compared with the pristine SWCNT, whereas the ISS for $-\mathrm{COOH}$ and $-\mathrm{CONH}_{2}$ functionalized groups has an almost identical value to that of the pristine SWCNT.

The interfacial binding energies of the functionalized CNTs for the cured epoxy systems (depicted in Fig. 5.19) are initially larger and presents more noticeable energy changes than that of the pristine CNTs as the pull-out process progresses. Similar to the SW defect, the initial larger interfacial binding energy is attributed to the stronger adsorption effect of the functionalized CNTs. Furthermore, the presence of a greater number of phenyl groups introduces additional molecular interactions, leading to a dramatic increase in the interfacial binding energy. It may be observed from Fig. 5.20 that a significant portion of the epoxy molecules is pulled out with the CNT during the pull-out process, indicating a stronger adhesion of the functionalized CNT with the cured epoxy matrix.

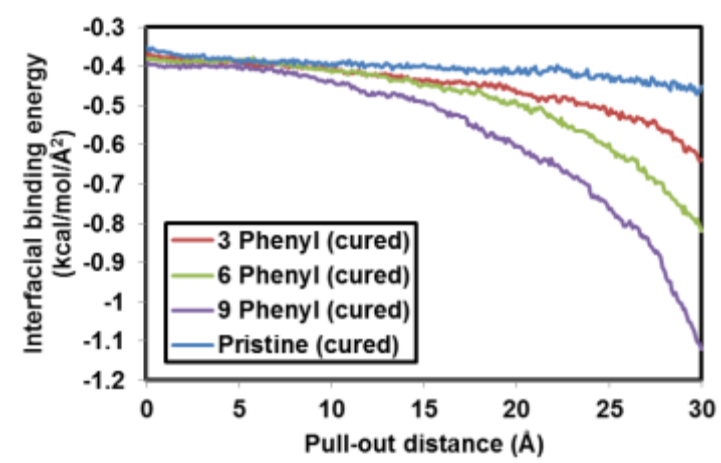

Fig. 5.19 Variations in interfacial binding energy between functionalized CNTs and cured epoxy matrix with pull-out distance.

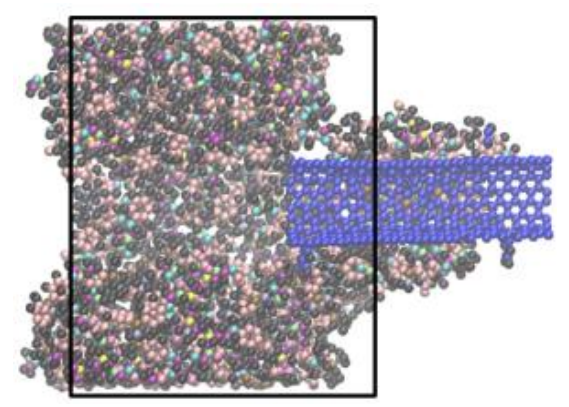

Fig. 5.20 Cross section of the pull-out process for 9 phenyl group functionalized CNT in cured epoxy matrix the at $z=30 \AA$. 


\subsection{Analysis of Compressive Load Simulation 5.2.1 Effect of Vacancy Defects upon freestanding SWCNTs}

This section focuses on exploring the effect of different vacancy defects on the buckling behavior of freestanding SWCNTs. Based on the foregoing definitions of Section 4.2.2, buckling strain and buckling load of the pristine SWCNTs are obtained in Table 5.1. We can see that the armchair SWCNT $(7,7)$ possesses very similar buckling capacity compared to the zigzag SWCNT $(12,0)$ due to the same length and diameter. However, the buckling strain of the armchair SWCNT $(9,9)$ is smaller than that of the armchair $\operatorname{SWCNT}(7,7)$ despite the same aspect ratio. The reverse is seen when the buckling load is considered. Our results are consistent with those reported by other researchers. For instance, Wang et al. [54] used MD simulations to study a broad range of armchair SWCNTs based on AIREBO potential. Their results showed that the buckling strain is in the range of 0.0131 to 0.0734 and the buckling load varies from $18.6 \mathrm{nN}$ to $86.8 \mathrm{nN}$. Zhang and coworkers $[87,91]$ also found that the nonlocal cylindrical shell model predicts comparable results compared with MD simulation results for SWCNTs with short aspect ratios $(\mathrm{L} / \mathrm{d}<8)$. The buckling strain can reach up to 0.1 when the aspect ratio is close to 5 . Zhang et al. [92] investigated the effect of chirality on the buckling behavior of SWCNTs. They reported that the influence of chirality can be neglected for SWCNTs with relatively larger chiral angles.

Table 5.1 Comparison of buckling behavior of freestanding defective SWCNTs against the buckling behavior of freestanding pristine SWCNTs.

\begin{tabular}{llcccc}
\hline $\begin{array}{l}\text { CNT } \\
\text { type }\end{array}$ & $\begin{array}{l}\text { CNT } \\
\text { configuration }\end{array}$ & $\begin{array}{l}\text { Buckling } \\
\text { strain }\end{array}$ & $\begin{array}{l}\text { \% reduction } \\
\text { w.r.t. pristine CNT }\end{array}$ & $\begin{array}{l}\text { Buckling load } \\
(\text { Kcal/mol-A) }\end{array}$ & $\begin{array}{c}\text { \% reduction } \\
\text { w.r.t. pristine CNT }\end{array}$ \\
\hline \multirow{6}{*}{ DA } & 0.095 & & $1276(89 \mathrm{nN})$ & \\
& 1A & 0.091 & 4.3 & 1208 & 5.3 \\
& 1B & 0.084 & 11.3 & 1178 & 7.7 \\
& 1C & 0.090 & 5.0 & 1140 & 10.7 \\
$(7,7)$ & 2B & 0.094 & 0.7 & 1134 & 11.1 \\
& 2C & 0.090 & 5.8 & 1215 & 4.8 \\
& 3A & 0.092 & 3.0 & 1094 & 14.2 \\
& 3B & 0.085 & 10.2 & 1145 & 11.5 \\
& 1SW-A & 0.093 & 2.5 & 1130 & 4.0 \\
& 1SW-B & 0.092 & 3.5 & 1224 & \\
& & & & & \\
$(9,9)$ & 0A & 0.091 & & $1560(108 \mathrm{nN})$ &
\end{tabular}




$\begin{array}{lcccc}\text { 1B } & 0.086 & 5.4 & 1575 & -0.9 \\ \text { 1C } & 0.087 & 4.3 & 1566 & -0.3 \\ \text { 2B } & 0.089 & 1.6 & 1513 & 3.0 \\ \text { 2C } & 0.087 & 3.7 & 1543 & 1.1 \\ \text { 3A } & 0.087 & 4.2 & 1418 & 9.1 \\ \text { 3B } & 0.086 & 4.8 & 1556 & 0.3 \\ \text { 1SW-A } & 0.085 & 6.3 & 1489 & 3.5 \\ \text { 1SW-B } & 0.089 & 1.4 & 1506 & \\ & & & & 1.8 \\ \text { 0A } & 0.094 & & 1222(85 \mathrm{nN}) & 6.0 \\ \text { 1A } & 0.090 & 4.6 & 1199 & 4.8 \\ \text { 1B } & 0.083 & 11.7 & 1104 & 3.9 \\ \text { 1C } & 0.087 & 7.3 & 1163 & 8.2 \\ \text { 2B } & 0.090 & 4.3 & 1174 & 8.6 \\ \text { 2C } & 0.091 & 3.6 & 1122 & 16.4 \\ \text { 3A } & 0.093 & 0.8 & 1117 & 6.8 \\ \text { 3B } & 0.085 & 9.7 & 1021 & 1138 \\ \text { 1SW-A } & 0.097 & -3.1 & 3.3 & \end{array}$

Next, we turn our attention to the effect of vacancy defects on the buckling behavior of freestanding CNTs. Fig. 5.21 shows the strain energy-strain and the force-displacement curves for the armchair $(7,7),(9,9)$ and zigzag $(12,0)$ defective SWCNTs. The buckling strain and buckling load obtained based on Fig. 5.21 are compared with the pristine SWCNTs and are tabulated in Table 5.1. The buckling modes of different defective SWCNTs are depicted in Fig. 4.4. It is interesting to observe in Fig. 4.4 that the buckling modes of various defective SWCNTs are similar to that of the pristine SWCNTs where one flattening is formed at the mid-length. In order to study the effect of missing atoms, only the symmetric vacancies (1A), (2B), and (3A) are analyzed here. It can also be seen from the results that the monovacancy (1A) has the lowest buckling strain, but increasing the number of missing atoms decreases the buckling load irrespective of the chirality of SWCNTs. It is easy to understand that monovacancy is superior to bivacancy and trivacancy because the defective area induced by the monovacancy is obviously smaller. However, by taking a closer look at the buckling shapes in Fig. 4.4, monovacancy tends to develop a flattening kink close to the defect, leading to excessive stress concentration. This makes the local buckling occur easily at the defect position. 


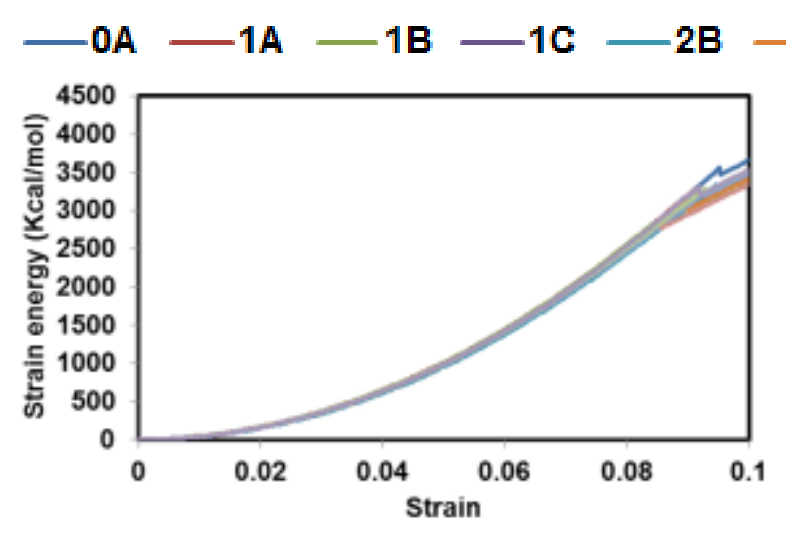

(a) $(7,7)$

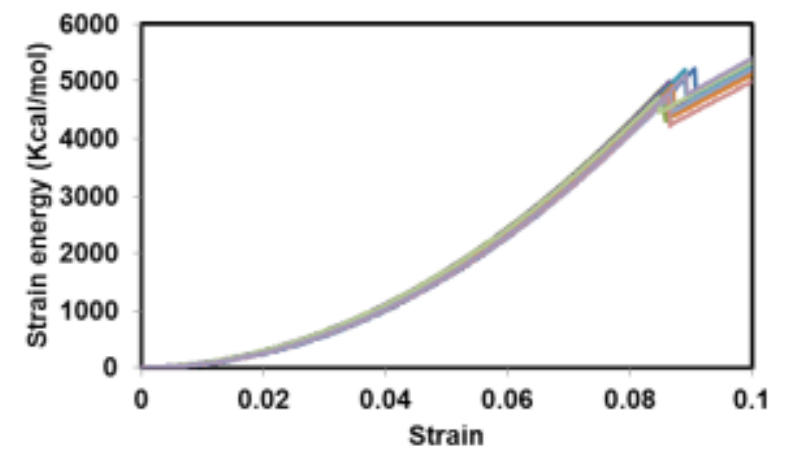

(c) $(9,9)$

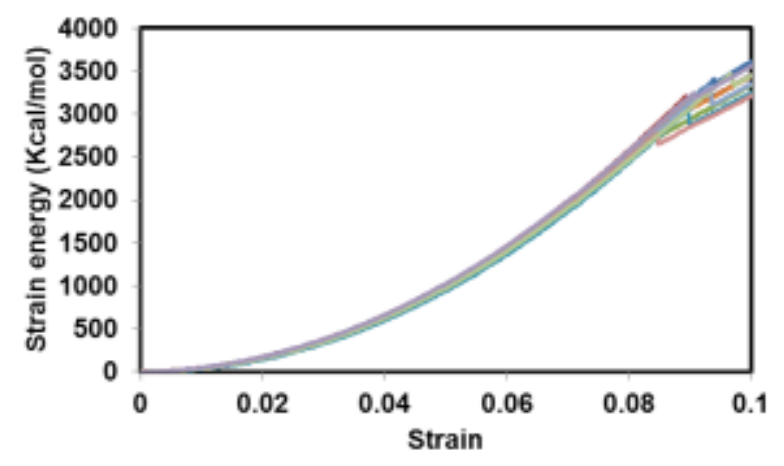

(e) $(12,0)$

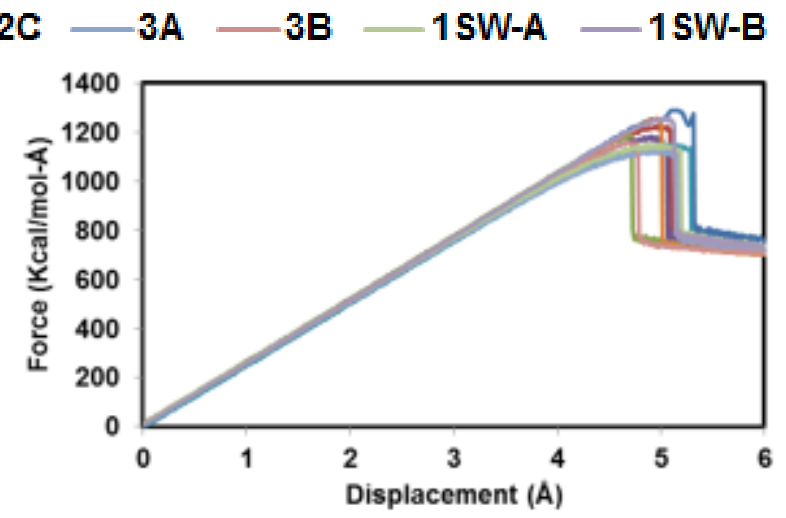

(b) $(7,7)$

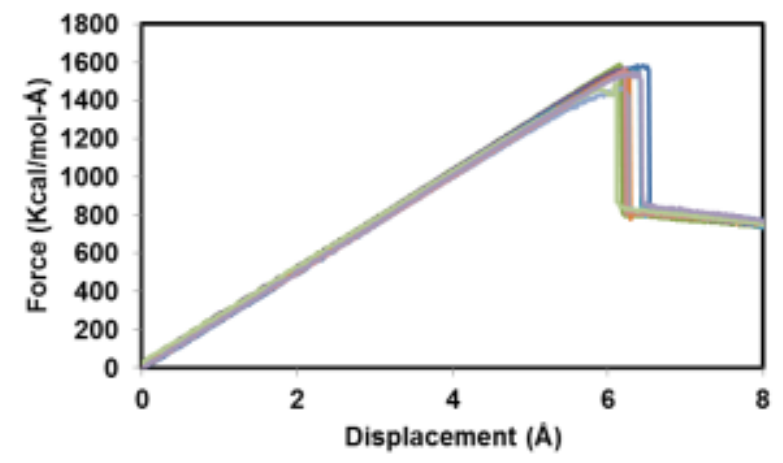

(d) $(9,9)$

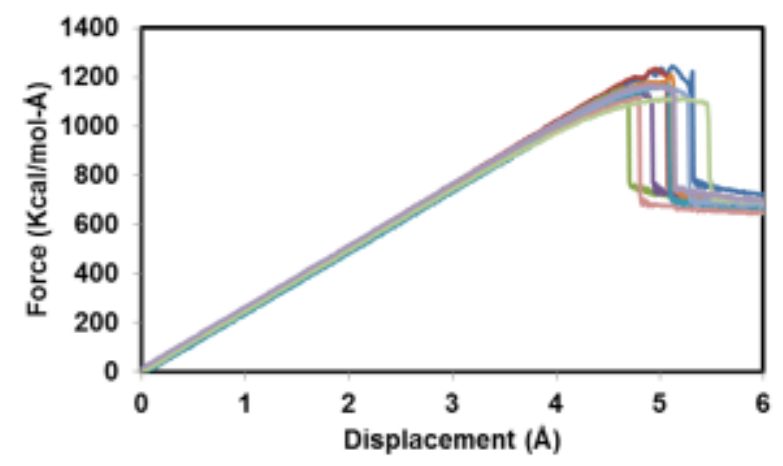

(f) $(12,0)$

Fig. 5.21 (a), (c) and (e) Strain energy-strain curves; (b), (d) and (f) force-displacement curves for different freestanding defective SWCNTs.

The vacancies (2B), (2C), (3A), and (3B) are considered next to study the effect of symmetric and asymmetric configurations on the buckling behavior. The results for armchair SWCNTs in Table 5.1 indicate that asymmetric vacancies, compared with symmetric vacancies, cause a higher reduction in the buckling strain but not in the buckling load. This can be explained by Fig. 4.4 that asymmetric vacancies develop flattening kinks near the defect, which expedites the 
occurrence of the buckling. Those kinks serve to withstand more loads and increase the buckling load accordingly. However, a difference can be observed between armchair and zigzag SWCNTs. For zigzag SWCNTs, asymmetric vacancies reduce both the buckling strain and buckling load, and the buckling behavior is most affected by trivacancies. This may be due to the special bond structure of zigzag CNTs.

The third focus here is on the effect of vacancy distribution on the buckling behavior of CNTs. Hence, we studied the nanotubes with vacancies (1B) and (1C). The results in Table 5.1 indicate that the vacancy (1B) has lower buckling strain but higher buckling load than the vacancy (1C). As shown in Fig. 4.4, this again occurs as a result of the positions of kinks relative to the defects. Since the monovacancies in (1B) locate exactly opposite to each other, the kinks tend to develop near the defects, leading to excessive stress concentration. This makes the vacancy (1B) easier to buckle than the vacancy (1C) but allows the vacancy (1B) to withstand higher loads for both armchair and zigzag SWCNTs.

\subsubsection{Effect of Missing Atoms upon Embedded SWCNTs}

In this section, we examine the effect of missing atoms on the buckling behavior of SWCNTs embedded in the epoxy matrix. Embedded pristine SWCNTs are initially taken as a reference to compare with freestanding pristine SWCNTs. Fig. 4.3(a) and 4.3(b) show the strain energy-strain and the force-displacement curves for the embedded pristine SWCNTs in comparison with pristine stand-alone SWCNTs. Table 5.2 shows the buckling strain and buckling load obtained from Fig. 4.3. Interestingly, the surrounding epoxy matrix significantly reduces resistance to buckling of SWCNTs by up to $43 \%$. The reason for the embedded CNTs to have lower resistance to buckling than their freestanding counterparts is the uneven atomic forces exerted by the surrounding matrix atoms. These atomic forces deform the CNTs unevenly, leading to easier occurrence of buckling. The buckled shapes in Fig. 5.22 show that the embedded pristine CNT displays only pure shell-like buckling modes, where two flattenings referred to as "fins" by Yakobson et al. [48] that are perpendicular to each other are formed. This is because the atomic forces exerted by the surrounding matrix atoms provide some form of confinement, preventing the CNTs from buckling sideway. The buckling shape transformation may also lead to the reduction in the buckling strain and buckling load. 
Table 5.2 Comparison of buckling behavior of embedded defective SWCNTs against the buckling behavior of embedded pristine SWCNTs.

\begin{tabular}{|c|c|c|c|c|c|}
\hline $\begin{array}{l}\text { Embedded } \\
\text { CNT type }\end{array}$ & $\begin{array}{l}\text { CNT } \\
\text { configuration }\end{array}$ & $\begin{array}{l}\text { Buckling } \\
\text { strain }\end{array}$ & $\begin{array}{l}\text { \% reduction } \\
\text { w.r.t. pristine } \\
\text { CNT }\end{array}$ & $\begin{array}{l}\text { Buckling load } \\
\text { (Kcal/mol- } \AA \text { ) }\end{array}$ & $\begin{array}{l}\% \text { reduction } \\
\text { w.r.t. pristine CNT }\end{array}$ \\
\hline \multirow{10}{*}{$(7,7)$} & $0 \mathrm{~A}$ & 0.071 & \multicolumn{3}{|c|}{$979(68 \mathrm{nN})$} \\
\hline & $1 \mathrm{~A}$ & 0.051 & 28.9 & 710 & 27.5 \\
\hline & $1 \mathrm{~B}$ & 0.050 & 30.0 & 676 & 30.9 \\
\hline & $1 \mathrm{C}$ & 0.053 & 25.0 & 629 & 35.7 \\
\hline & $2 B$ & 0.054 & 24.4 & 712 & 27.3 \\
\hline & $2 \mathrm{C}$ & 0.047 & 34.5 & 629 & 35.7 \\
\hline & $3 \mathrm{~A}$ & 0.057 & 20.1 & 756 & 22.7 \\
\hline & $3 B$ & 0.043 & 40.2 & 576 & 41.2 \\
\hline & 1SW-A & 0.059 & 17.0 & 784 & 19.9 \\
\hline & 1SW-B & 0.052 & 27.4 & 690 & 29.6 \\
\hline \multirow{10}{*}{$(9,9)$} & $0 \mathrm{~A}$ & 0.052 & \multicolumn{3}{|c|}{$912(63 \mathrm{nN})$} \\
\hline & $1 \mathrm{~A}$ & 0.043 & 18.4 & 733 & 19.6 \\
\hline & $1 \mathrm{~B}$ & 0.042 & 19.4 & 733 & 19.6 \\
\hline & $1 \mathrm{C}$ & 0.049 & 7.3 & 768 & 15.8 \\
\hline & $2 \mathrm{~B}$ & 0.044 & 15.9 & 771 & 15.4 \\
\hline & $2 \mathrm{C}$ & 0.045 & 15.1 & 762 & 16.4 \\
\hline & $3 \mathrm{~A}$ & 0.044 & 15.5 & 631 & 30.8 \\
\hline & $3 B$ & 0.043 & 17.9 & 722 & 20.8 \\
\hline & $1 \mathrm{SW}-\mathrm{A}$ & 0.048 & 7.6 & 866 & 5.1 \\
\hline & 1SW-B & 0.042 & 19.5 & 749 & 17.9 \\
\hline \multirow{10}{*}{$(12,0)$} & $0 \mathrm{~A}$ & 0.070 & \multicolumn{3}{|c|}{$959(67 \mathrm{nN})$} \\
\hline & $1 \mathrm{~A}$ & 0.053 & 24.9 & 711 & 25.9 \\
\hline & $1 \mathrm{~B}$ & 0.042 & 39.7 & 564 & 41.2 \\
\hline & $1 \mathrm{C}$ & 0.053 & 24.1 & 680 & 29.1 \\
\hline & $2 \mathrm{~B}$ & 0.052 & 25.9 & 678 & 29.3 \\
\hline & $2 \mathrm{C}$ & 0.049 & 30.6 & 614 & 36.0 \\
\hline & $3 \mathrm{~A}$ & 0.052 & 26.2 & 656 & 31.6 \\
\hline & $3 B$ & 0.046 & 35.1 & 605 & 36.9 \\
\hline & 1SW-A & 0.056 & 20.7 & 750 & 21.8 \\
\hline & 1SW-B & 0.051 & 26.9 & 699 & 27.2 \\
\hline
\end{tabular}




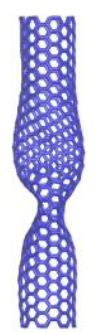

OA

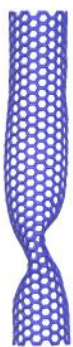

$O A$

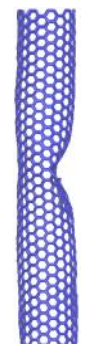

$1 \mathrm{~A}$

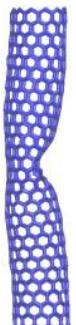

$1 \mathrm{~A}$

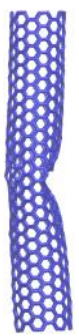

1B
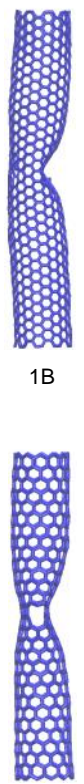

1B
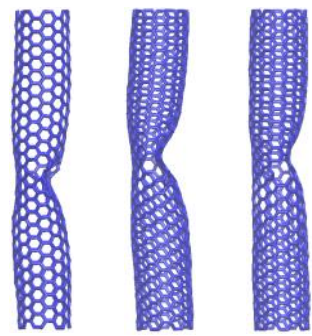

2C

(a) $(7,7)$
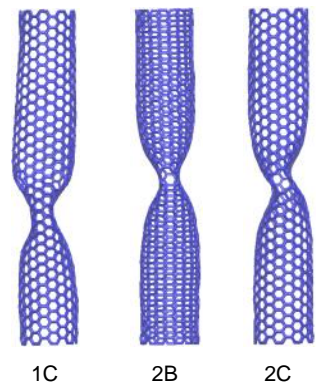

2C

(b) $(9,9)$

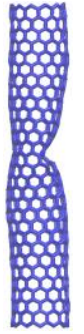

$1 \mathrm{C}$
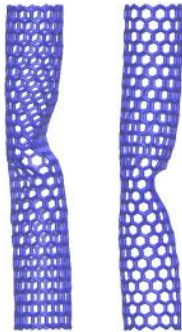

2C
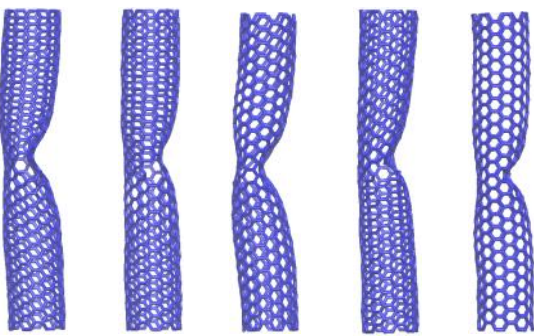

1SW-A
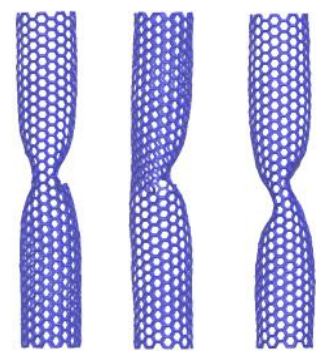

1SW-A

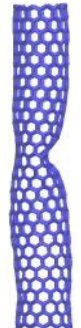

(c) $(12,0)$
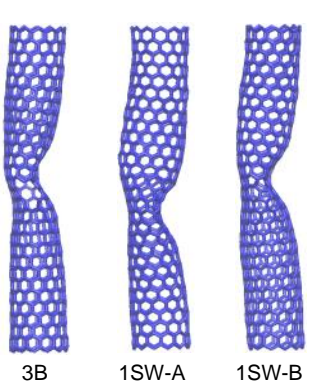

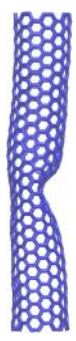

1SW-B

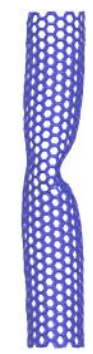

1SW-B

Fig. 5.22 Buckling mode shapes of the embedded SWCNTs (a), (7, 7), (b) $(9,9)$ and (c) $(12,0)$.

We now proceed to study the buckling of nanotubes with different missing atoms (1A), (2B), and (3A) embedded in the epoxy matrix. Fig. 5.23 shows the strain energy-strain and the loaddisplacement plots for the defective embedded nanotubes discussed above. As demonstrated in Table 5.2, both the buckling strain and buckling load increase as the number of missing atoms increases. The maximum reductions in the buckling strain and buckling load are $29 \%$ and $31 \%$, respectively, which is higher than what we observed in freestanding defective armchair SWCNTs. This may be explained by the buckled shapes depicted in Fig. 5.22; the first buckling mode occurs only on one side of the SWCNTs near the missing atoms, which are neither beamlike nor shell-like buckling modes. These buckled modes depicted in Fig. 5.22 result in excessive stress concentration for monovacancies, leading to a minima of both the buckling strain and the buckling load. However, an exception can be observed for the $(9,9)$ SWCNT with the trivacancy (3A) which has the lowest buckling load. A closer look at the buckled shape of (3A) reveals that 
it displays a shell-like buckling mode due to its longer nanotube. This may contribute to a reduction in the buckling load. In contrast to the embedded defective armchair SWCNTs, Table 5.2 shows that both the buckling strain and buckling load of the embedded defective zigzag SWCNTs decrease with increasing number of missing atoms. This reveals that more missing atoms in zigzag SWCNTs have more negative influence on the buckling behavior despite similar buckling shapes to the embedded defective armchair SWCNTs.

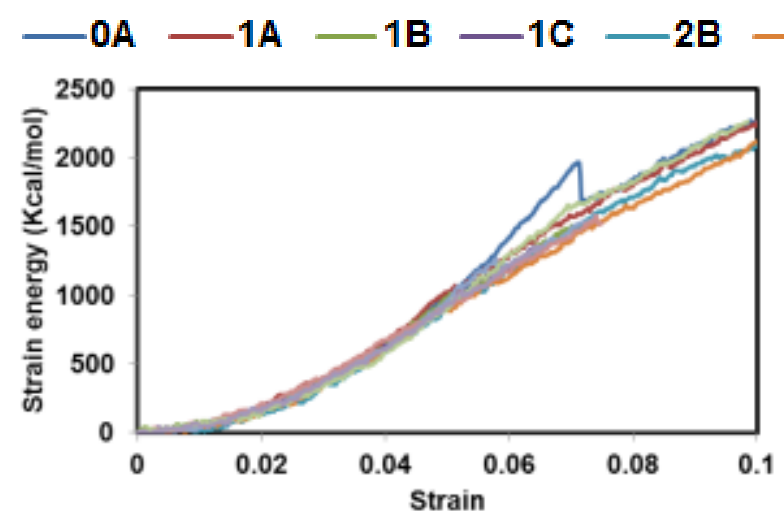

(a) $(7,7)$

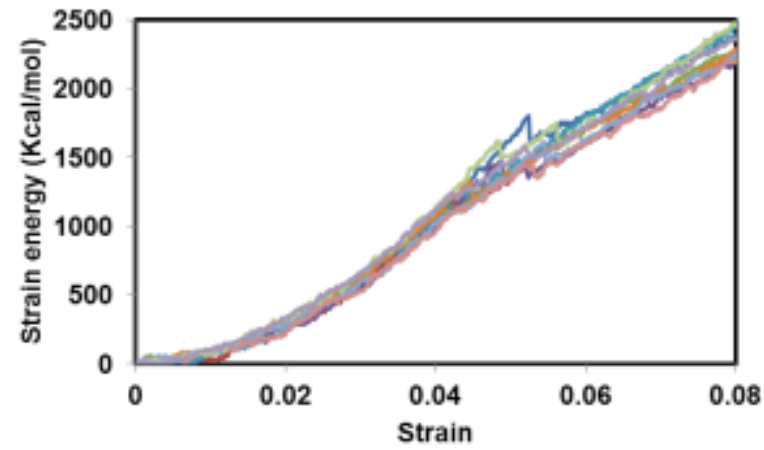

(c) $(9,9)$

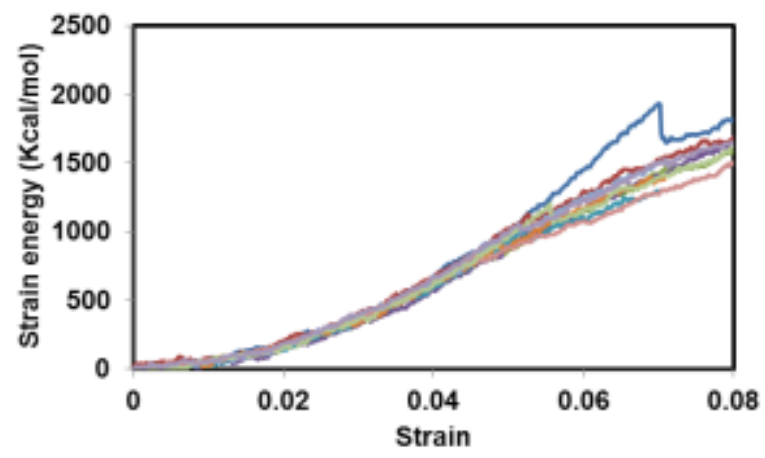

(e) $(12,0)$

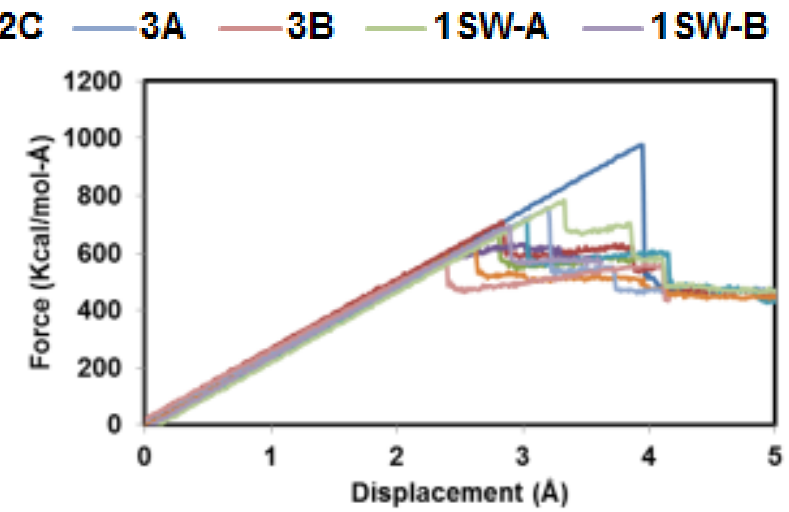

(b) $(7,7)$

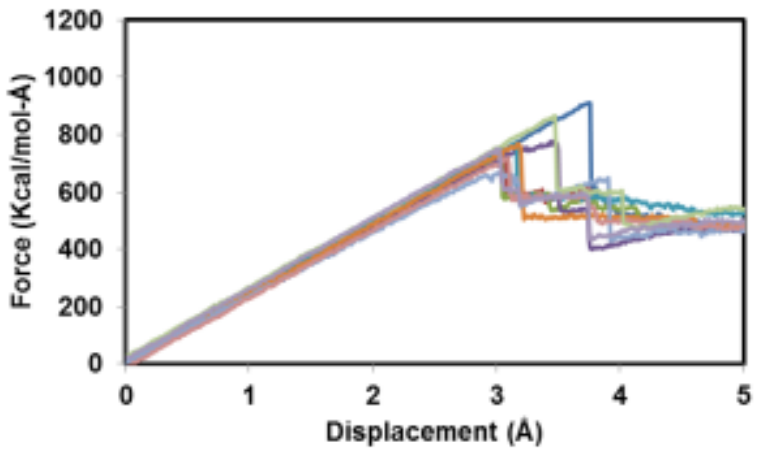

(d) $(9,9)$

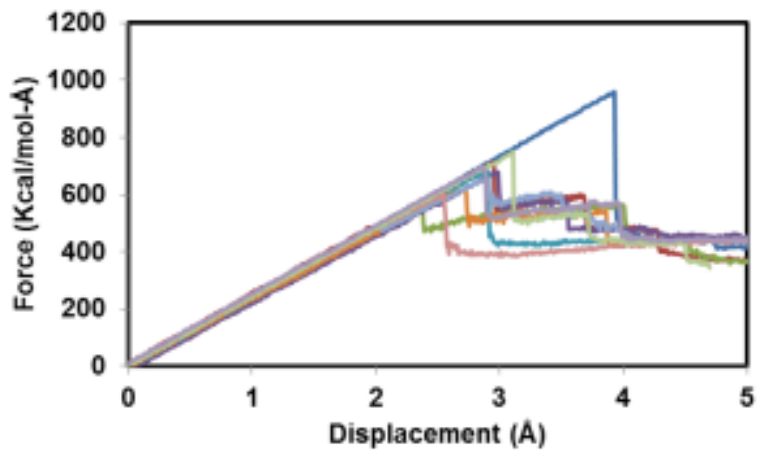

(f) $(12,0)$

Fig. 5.23 (a), (c) and (e) Strain energy-strain curves; (b), (d) and (f) force-displacement curves for different embedded defective SWCNTs. 


\subsubsection{Effect of Vacancy Symmetry and Distribution upon Embedded SWCNTs}

First, let us focus attention on the effect of symmetric and asymmetric vacancy configurations (2B), (2C), (3A) and (3B) on the buckling behavior of embedded SWCNTs. It can be seen in Table 5.2 that asymmetric vacancies generally reduce the buckling behavior of both armchair and zigzag SWCNTs when compared with symmetric vacancies. This is different from corresponding freestanding armchair nanotubes, where the buckling load is increased due to the presence of kinks in asymmetric vacancies. Compared with the symmetric vacancies, asymmetric vacancies further reduce the buckling strain and buckling load by $5 \%$ to $20 \%$. The reason for the discrepancies may be because embedded defective SWCNTs display different buckling modes in Fig. 5.22, where the nanotubes only narrow at one side while keep their cylindrical shapes at the other side. The position of the narrowing is close to the vacancies. However, due to the longer length in $(9,9)$ nanotubes, the buckling behavior of the bivacancies (2B) and (2C) is very similar to each other. They tend to buckle in a shell-like way similar to the embedded pristine SWCNTs.

Second, let us devote our attention to the effect of vacancy distribution (1B) and (1C) on the buckling behavior of embedded SWCNTs. Unlike the case of freestanding SWCNTs, both the buckling strain and buckling load of the SWCNTs containing the vacancy (1C) (see Table 5.2) are increased when compared with those of the SWCNTs containing the vacancy (1B) irrespective of the chirality of SWCNTs. This may be due to the different buckling modes presented in Fig. 5.22, which are similar to the previously investigated embedded SWCNTs. It is worth noting that there exists an anomaly for the buckling load of the $(7,7)$ SWCNT with the vacancy (1C). It is clear from Fig. 5.23(b) that the $(7,7)$ SWCNT with the vacancy (1C) does not show an abrupt drop in the load-displacement curve. We can see that the vacancy (1C) in the (7, 7) SWCNT helps to delay the occurrence of buckling, which increases the buckling strain, although the buckling load is still relatively low.

\subsubsection{Effect of SW defects upon Freestanding and Embedded SWCNTs}

As shown in Table 5.1, the SW defects reduce the buckling capacity of SWCNTs in general. Similar to the vacancy symmetry of freestanding SWCNTs, asymmetry results in lower buckling strain but generally requires higher buckling load irrespective of the chirality of SWCNTs. It is noted in Fig. 4.4 that kinks tend to locate close to the asymmetric SW defect (1SW-B). This 
leads to excessive stress concentration, which expedites the occurrence of the buckling, and the kinks help to withstand more loads, increasing the buckling load.

The second part of this section studies the SW defects (1SW-A) and (1SW-B) of embedded SWCNTs. The embedded SWCNTs containing asymmetric SW defects (1SW-B) have lower buckling strain and buckling load (see Table 5.2) regardless of the chirality of SWCNTs. The maximum decreases in the buckling strain and buckling load are $22 \%$ and $30 \%$, respectively, which are higher than the reduction found in their freestanding counterparts. The buckling modes for the embedded SW defective SWCNTs are similar to other embedded SWCNTs. Moreover, if we compare the buckling behavior of SW defective SWCNTs with vacancy defective SWCNTs in Table 5.2, it is evident that the SW defects (1SW-A) have the least effect on the buckling behavior of embedded SWCNTs. By contrast, the degradation effect of embedded SW defective SWCNTs (1SW-B) is very similar to the corresponding embedded vacancy defective SWCNTs. 


\section{Chapter 6 \\ Conclusions and Future Work}

Summary: In this final chapter, we provide a summary of the major findings of the research conducted and identify the original contributions of the thesis. Furthermore, we discuss the limitations of the investigation and provide a brief description of the key areas that would benefit from additional future work.

\subsection{General Conclusions of Pull-out Simulation}

In the pull-out simulation, we studied the influence of the most common defects in CNTs upon the ISS of nano-reinforced thermoset epoxy before and after cross-linking. Specifically, we examined the effect of vacancy, adatom, and SW in CNTs upon the ISS of defective CNTreinforced epoxy polymer. We also reviewed the effect of functionalization upon the ISS of the nanocomposite. The main conclusions of the pull-out studies are summarized as follows:

(i) Among all the defects and functionalization, the influence of vacancy defects on the ISS was found to be significant. Moreover, the influence of the same number of missing atoms on the ISS was found to be almost identical.

(ii) Adatom defects have only marginal effect on the ISS. The ISS increased for the cured epoxy systems as the number of SW defects increases.

(iii) In the case of phenyl groups, the ISS of the cured systems increases due to the additional vdW interactions generated as a result of functionalization.

(iv) Uncured epoxy systems have lower ISS than cured ones, and defects and functionalization do not affect the ISS very much.

Although the defective and functionalized CNTs exhibit lower mechanical strength compared with the pristine CNTs, our results reveal that SW defects and functionalization in cured systems can significantly improve the CNT-matrix load transferability. To sum up our findings, the defective and functionalized CNTs in cured epoxy systems can lead to increased ISS and improved load transferability. However, the presence of defects in the CNTs and epoxy materials with a lower degree of conversion would ultimately result in decrease in the effective elastic properties of the nanocomposites. A trade-off between the degradation in the mechanical 
properties of nanocomposites and the improvement in the ISS of the resulting multifunctional nanocomposite systems should be carefully considered and addressed.

\subsection{General Conclusions of Compressive Load Simulation}

The aim of the compressive load simulation is to conduct a quantitative analysis of the degradation of buckling strain and buckling load due to various types of defects (vacancy and SW defect) using MD simulations. Both freestanding and embedded SWCNTs with different sizes and chiralities $((7,7),(9,9)$, and $(12,0))$ were simulated and the results were compared with each other to understand the effect of epoxy matrix. To understand the factors affecting the degree of degradation of buckling strain and buckling load, we investigated several defect configurations including the number of missing atoms, symmetry, and vacancy distribution. The main conclusions of the compressive load studies are summarized as follows:

(i) Defects generally have the same effect on the buckling strain of freestanding and embedded SWCNTs. However, compared with those of freestanding SWCNTs, the buckling strain and buckling load of embedded SWCNTs are reduced when SWCNTs are confined in an epoxy matrix.

(ii) Increasing number of missing atoms generally reduces the buckling load of freestanding SWCNTs; however, more number of missing atoms increases the buckling load of embedded armchair SWCNTs because the compressive force is partly supported by the kinks developed during buckling.

(iii) The buckling load of freestanding SWCNTs is increased by asymmetric vacancy and SW defects, but the same defects decrease the buckling load of embedded SWCNTs due to different buckling modes.

(iv) Freestanding SWCNTs containing asymmetric vacancy distribution have higher buckling load because of the positions of kinks relative to the defects, but the opposite is true for embedded SWCNTs in general.

Defects are unavoidable in CNTs during the synthesis and manufacturing of CNT-reinforced nanocomposites. Different buckling behaviors are seen between freestanding and embedded CNTs in the presence of various defects. We cannot rely on defect studies focusing only on the 
buckling of freestanding CNTs when designing CNT-reinforced nanocomposites. It is hoped that the findings in this study could contribute to deciding the expected strength from a certain sample of CNT-reinforced nanocomposites, if the degradation of buckling strain and buckling load due to different defects is known. Therefore, this study is believed to be helpful in designing multifunctional nanocomposites.

\subsection{Thesis Original Contributions}

The main contributions of the current work can be summarized as follows:

(i) Developed accurate MD models to capture the effect of matrix molecules (e.g., crosslinked structures and aromatic rings) on embedded CNTs for pull-out and compressive load simulations.

(ii) Conducted comprehensive studies on the effect of various defects such as vacancy, SW defect, carbon adatom, and phenyl functional group on the ISS of CNTreinforced composites with different degrees of conversion.

(iii) Carried out systematic investigations on the effect of various defects such as vacancy and SW defect with different configurations and distributions on the buckling behavior of CNT-reinforced composites.

\subsection{Future Work}

The following areas are worthy of consideration in future research projects:

(i) The model only considers uniformly distributed and straight CNTs of constant aspect ratio. In order to better correlate the results to experimental measurements, the model must be extended to consider agglomeration, CNT curvature, and interface cracks.

(ii) Since the CVFF does not describe the bond order change of the valence systems, migration and reconstruction of the defects cannot be considered in this research. Also, experimentally observed cross-links between CNTs and matrices cannot be modeled. Therefore, it is important to develop more advanced forcefield which allows bond building and breaking.

(iii) Extend the model to investigate MWCNTs and other matrix materials and develop multiscale models to consider bulk nanocomposites. 


\section{References}

[1] A. Vlot, Glare: History of the Development of a New Aircraft Material: Springer Netherlands, 2007.

[2] A. Vlot and J. W. Gunnink, Fibre Metal Laminates: An Introduction: Springer Netherlands, 2011.

[3] D. Brosius. (2007). Boeing 787 Update. Available: http://www.compositesworld.com/articles/boeing-787update

[4] J. Hale. (2006). Boeing 787 From the Ground Up. Available: http://www.boeing.com/commercial/aeromagazine/articles/qtr_4_06/AERO_Q406_article4.pdf

[5] E. Udd, Fiber Optic Smart Structures: Wiley, 1995.

[6] S. Iijima, "Helical microtubules of graphitic carbon," Nature, vol. 354, pp. 56-58, 1991.

[7] Q. H. Wang, A. Setlur, J. Lauerhaas, J. Dai, E. Seelig, and R. P. Chang, "A nanotube-based field-emission flat panel display," Applied Physics Letters, vol. 72, pp. 2912-2913, 1998.

[8] Q. B. Zheng, D. Xia, Q. Z. Xue, K. Y. Yan, X. L. Gao, and Q. Li, "Computational analysis of effect of modification on the interfacial characteristics of a carbon nanotube-polyethylene composite system," Applied Surface Science, vol. 255, pp. 3534-3543, 2009.

[9] Q. Zheng, Q. Xue, K. Yan, X. Gao, Q. Li, and L. Hao, "Effect of chemisorption on the interfacial bonding characteristics of carbon nanotube-polymer composites," Polymer, vol. 49, pp. 800-808, 2008.

[10] S. Yang, J. Choi, and M. Cho, "Intrinsic defect-induced tailoring of interfacial shear strength in CNT/polymer nanocomposites," Composite Structures, vol. 127, pp. 108-119, 2015.

[11] J. M. Wernik and S. A. Meguid, "Multiscale modeling of the nonlinear response of nano-reinforced polymers," Acta Mechanica, vol. 217, pp. 1-16, 2011.

[12] C. Jang, T. E. Lacy, S. R. Gwaltney, H. Toghiani, and C. U. Pittman Jr, "Interfacial shear strength of cured vinyl ester resin-graphite nanoplatelet from molecular dynamics simulations," Polymer, vol. 54, pp. 32823289, 2013.

[13] S. C. Chowdhury, T. Okabe, and M. Nishikawa, "Effects of vacancy defects on the interfacial shear strength of carbon nanotube reinforced polymer composite," Journal of Nanoscience and Nanotechnology, vol. 10, pp. 739-745, 2010.

[14] J. M. Wernik and S. A. Meguid, "Recent Developments in Multifunctional Nanocomposites Using Carbon Nanotubes," Applied Mechanics Reviews, vol. 63, pp. 050801-050801, 2011.

[15] S. Frankland, A. Caglar, D. Brenner, and M. Griebel, "Molecular simulation of the influence of chemical cross-links on the shear strength of carbon nanotube-polymer interfaces," The Journal of Physical Chemistry $B$, vol. 106, pp. 3046-3048, 2002.

[16] J. C. Meyer, C. Kisielowski, R. Erni, M. D. Rossell, M. Crommie, and A. Zettl, "Direct imaging of lattice atoms and topological defects in graphene membranes," Nano Letters, vol. 8, pp. 3582-3586, 2008.

[17] F. Banhart, J. Kotakoski, and A. V. Krasheninnikov, "Structural Defects in Graphene," ACS Nano, vol. 5, pp. 26-41, 2011.

[18] C. Bower, R. Rosen, L. Jin, J. Han, and O. Zhou, "Deformation of carbon nanotubes in nanotube-polymer composites," Applied Physics Letters, vol. 74, pp. 3317-3319, 1999. 
[19] S.-h. Hwang, D. S. Bang, K. H. Yoon, and Y.-B. Park, Smart materials and structures based on carbon nanotube composites: INTECH Open Access Publisher, 2011.

[20] Z. Xia, L. Riester, W. A. Curtin, H. Li, B. W. Sheldon, J. Liang, et al., "Direct observation of toughening mechanisms in carbon nanotube ceramic matrix composites," Acta Materialia, vol. 52, pp. 931-944, 2004.

[21] C. A. Cooper, S. R. Cohen, A. H. Barber, and H. D. Wagner, "Detachment of nanotubes from a polymer matrix," Applied Physics Letters, vol. 81, pp. 3873-3875, 2002.

[22] A. H. Barber, S. R. Cohen, and H. D. Wagner, "Measurement of carbon nanotube-polymer interfacial strength," Applied Physics Letters, vol. 82, pp. 4140-4142, 2003.

[23] A. H. Barber, S. R. Cohen, A. Eitan, L. S. Schadler, and H. D. Wagner, "Fracture transitions at a carbon nanotube/polymer interface," Advanced Materials, vol. 18, pp. 83-87, 2006.

[24] D. Qian, E. C. Dickey, R. Andrews, and T. Rantell, "Load transfer and deformation mechanisms in carbon nanotube-polystyrene composites," Applied Physics Letters, vol. 76, pp. 2868-2870, 2000.

[25] S. A. Meguid and Y. Sun, "On the tensile and shear strength of nano-reinforced composite interfaces," Materials \& Design, vol. 25, pp. 289-296, 2004.

[26] X. Xu, M. M. Thwe, C. Shearwood, and K. Liao, "Mechanical properties and interfacial characteristics of carbon-nanotube-reinforced epoxy thin films," Applied Physics Letters, vol. 81, pp. 2833-2835, 2002.

[27] H. Wagner, O. Lourie, Y. Feldman, and R. Tenne, "Stress-induced fragmentation of multiwall carbon nanotubes in a polymer matrix," Applied Physics Letters, vol. 72, pp. 188-190, 1998.

[28] X. L. Gao and K. Li, "A shear-lag model for carbon nanotube-reinforced polymer composites," International Journal of Solids and Structures, vol. 42, pp. 1649-1667, 2005.

[29] H. L. Cox, "The elasticity and strength of paper and other fibrous materials," British Journal of Applied Physics, vol. 3, p. 72, 1952.

[30] K. Li and S. Saigal, "Micromechanical modeling of stress transfer in carbon nanotube reinforced polymer composites," Materials Science and Engineering: A, vol. 457, pp. 44-57, 2007.

[31] J. Gou, Z. Liang, C. Zhang, and B. Wang, "Computational analysis of effect of single-walled carbon nanotube rope on molecular interaction and load transfer of nanocomposites," Composites Part B: Engineering, vol. 36, pp. 524-533, 2005.

[32] J. H. Gou, B. Minaie, B. Wang, Z. Y. Liang, and C. Zhang, "Computational and experimental study of interfacial bonding of single-walled nanotube reinforced composites," Computational Materials Science, vol. 31, pp. 225-236, 2004.

[33] J. Q. Liu, T. Xiao, K. Liao, and P. Wu, "Interfacial design of carbon nanotube polymer composites: a hybrid system of noncovalent and covalent functionalizations," Nanotechnology, vol. 18, p. 165701, 2007.

[34] Y. Li, Y. Liu, X. Peng, C. Yan, S. Liu, and N. Hu, "Pull-out simulations on interfacial properties of carbon nanotube-reinforced polymer nanocomposites," Computational Materials Science, vol. 50, pp. 1854-1860, 2011.

[35] K. Sharma, K. Sen Kaushalyayan, and M. Shukla, "Pull-out simulations of interfacial properties of amine functionalized multi-walled carbon nanotube epoxy composites," Computational Materials Science, vol. 99, pp. 232-241, 2015. 
[36] Q. L. Xiong and S. A. Meguid, "Atomistic investigation of the interfacial mechanical characteristics of carbon nanotube reinforced epoxy composite," European Polymer Journal, 2015.

[37] A. Alian, S. Kundalwal, and S. Meguid, "Interfacial and mechanical properties of epoxy nanocomposites using different multiscale modeling schemes," Composite Structures, vol. 131, pp. 545-555, 2015.

[38] J. M. Wernik, B. J. Cornwell-Mott, and S. A. Meguid, "Determination of the interfacial properties of carbon nanotube reinforced polymer composites using atomistic-based continuum model," International Journal of Solids and Structures, vol. 49, pp. 1852-1863, 2012.

[39] M. B. Nardelli, B. I. Yakobson, and J. Bernholc, "Brittle and ductile behavior in carbon nanotubes," Physical Review Letters, vol. 81, p. 4656, 1998.

[40] J.-C. Charlier, "Defects in carbon nanotubes," Accounts of Chemical Research, vol. 35, pp. 1063-1069, 2002.

[41] P. G. Collins, "Defects and disorder in carbon nanotubes," Oxford Handbook of Nanoscience and Technology: Frontiers and Advances, 2010.

[42] Z. W. Pan, S. S. Xie, L. Lu, B. H. Chang, L. F. Sun, W. Y. Zhou, et al., "Tensile tests of ropes of very long aligned multiwall carbon nanotubes," Applied Physics Letters, vol. 74, pp. 3152-3154, 1999.

[43] H. Dai, J. H. Hafner, A. G. Rinzler, D. T. Colbert, and R. E. Smalley, "Nanotubes as nanoprobes in scanning probe microscopy," Nature, vol. 384, pp. 147-150, 1996.

[44] S. Iijima, C. Brabec, A. Maiti, and J. Bernholc, "Structural flexibility of carbon nanotubes," The Journal of Chemical Physics, vol. 104, pp. 2089-2092, 1996.

[45] E. W. Wong, P. E. Sheehan, and C. M. Lieber, "Nanobeam mechanics: elasticity, strength, and toughness of nanorods and nanotubes," Science, vol. 277, pp. 1971-1975, 1997.

[46] O. Lourie, D. Cox, and H. Wagner, "Buckling and collapse of embedded carbon nanotubes," Physical Review Letters, vol. 81, p. 1638, 1998.

[47] L. Schadler, S. Giannaris, and P. Ajayan, "Load transfer in carbon nanotube epoxy composites," Applied Physics Letters, vol. 73, pp. 3842-3844, 1998.

[48] B. I. Yakobson, C. J. Brabec, and J. Bernholc, "Nanomechanics of Carbon Tubes: Instabilities beyond Linear Response," Physical Review Letters, vol. 76, pp. 2511-2514, 1996.

[49] C. Ru, "Effect of van der Waals forces on axial buckling of a double-walled carbon nanotube," Journal of Applied Physics, vol. 87, pp. 7227-7231, 2000.

[50] C. Y. Wang, C. Q. Ru, and A. Mioduchowski, "Axially compressed buckling of pressured multiwall carbon nanotubes," International Journal of Solids and Structures, vol. 40, pp. 3893-3911, 2003.

[51] Y. Y. Zhang, C. M. Wang, and V. B. C. Tan, "Buckling of carbon nanotubes at high temperatures," Nanotechnology, vol. 20, 2009.

[52] K. Talukdar, R. Agrawala, and A. K. Mitra, "Dependence of mechanical characteristics and the fracture and buckling behavior of single-walled carbon nanotubes on their geometry," New Carbon Materials, vol. 26, pp. 408-416, 2011.

[53] B. Motevalli, A. Montazeri, J. Z. Liu, and H. Rafii-Tabar, "Comparison of continuum-based and atomisticbased modeling of axial buckling of carbon nanotubes subject to hydrostatic pressure," Computational Materials Science, vol. 79, pp. 619-626, 2013. 
[54] C. Wang, A. R. Chowdhury, S. Koh, and Y. Zhang, "Molecular dynamics simulation and continuum shell model for buckling analysis of carbon nanotubes," in Modeling of Carbon Nanotubes, Graphene and their Composites, ed: Springer International Publishing, 2014, pp. 239-273.

[55] Y. Y. Zhang, C. M. Wang, and V. B. C. Tan, "Buckling of multiwalled carbon nanotubes using Timoshenko beam theory," Journal of Engineering Mechanics, vol. 132, pp. 952-958, 2006.

[56] C. Ru, "Axially compressed buckling of a doublewalled carbon nanotube embedded in an elastic medium," Journal of the Mechanics and Physics of Solids, vol. 49, pp. 1265-1279, 2001.

[57] S. Timoshenko, Theory of elastic stability: McGraw-Hill, 1961.

[58] S. Kitipornchai, X. He, and K. Liew, "Buckling analysis of triple-walled carbon nanotubes embedded in an elastic matrix," Journal of Applied Physics, vol. 97, 2005.

[59] K. M. Liew, X. Q. He, and S. Kitipornchai, "Buckling characteristics of embedded multi-walled carbon nanotubes," Proceedings of the Royal Society a-Mathematical Physical and Engineering Sciences, vol. 461, pp. 3785-3805, 2005.

[60] N. Chandra and S. Namilae, "Tensile and compressive behavior of carbon nanotubes: effect of functionalization and topological defects," Mechanics of Advanced Materials and Structures, vol. 13, pp. 115-127, 2006.

[61] H. Xin, Q. Han, and X.-H. Yao, "Buckling and axially compressive properties of perfect and defective singlewalled carbon nanotubes," Carbon, vol. 45, pp. 2486-2495, 2007.

[62] X. Hao, H. Qiang, and X. Yao, "Buckling of defective single-walled and double-walled carbon nanotubes under axial compression by molecular dynamics simulation," Composites Science and Technology, vol. 68, pp. 1809-1814, 2008.

[63] Y. Y. Zhang, Y. Xiang, and C. M. Wang, "Buckling of defective carbon nanotubes," Journal of Applied Physics, vol. 106, 2009.

[64] D. D. T. K. Kulathunga, K. K. Ang, and J. N. Reddy, "Molecular dynamics analysis on buckling of defective carbon nanotubes," Journal of Physics-Condensed Matter, vol. 22, 2010.

[65] A. R. Ranjbartoreh and G. Wang, "Effect of Topological Defects on Buckling Behavior of Single-walled Carbon Nanotube," Nanoscale Research Letters, vol. 6, 2011.

[66] V. Parvaneh, M. Shariati, and A. M. M. Sabed, "Investigation of vacancy defects effects on the buckling behavior of SWCNTs via a structural mechanics approach," European Journal of Mechanics a-Solids, vol. 28, pp. 1072-1078, 2009.

[67] R. H. Poelma, H. Sadeghian, S. Koh, and G. Q. Zhang, "Effects of single vacancy defect position on the stability of carbon nanotubes," Microelectronics Reliability, vol. 52, pp. 1279-1284, 2012.

[68] Q. Cheng, X. X. Wang, and N. G. Ni, "Molecular dynamics simulation for compressive mechanics properties of SWCNT with random distributed vacancies," in Nanoscience and Technology, Pts 1 and 2. vol. 121-123, C. Bai, S. Xie, and X. Zhu, Eds., ed, 2007, pp. 1161-1164.

[69] K. Suenaga, H. Wakabayashi, M. Koshino, Y. Sato, K. Urita, and S. Iijima, "Imaging active topological defects in carbon nanotubes," Nat Nano, vol. 2, pp. 358-360, 2007.

[70] A. Hashimoto, K. Suenaga, A. Gloter, K. Urita, and S. Iijima, "Direct evidence for atomic defects in graphene layers," Nature, vol. 430, pp. 870-873, 2004. 
[71] L. Liu, A. H. Barber, S. Nuriel, and H. D. Wagner, "Mechanical properties of functionalized single-walled carbon-nanotube/poly(vinyl alcohol) nanocomposites," Advanced Functional Materials, vol. 15, pp. 975-980, 2005.

[72] Z. Spitalsky, D. Tasis, K. Papagelis, and C. Galiotis, "Carbon nanotube-polymer composites: Chemistry, processing, mechanical and electrical properties," Progress in Polymer Science, vol. 35, pp. 357-401, 2010.

[73] E. T. Thostenson, C. Li, and T.-W. Chou, "Nanocomposites in context," Composites Science and Technology, vol. 65, pp. 491-516, 2005.

[74] T. Xiao, J. Liu, and H. Xiong, "Effects of different functionalization schemes on the interfacial strength of carbon nanotube polyethylene composite," Acta Mechanica Solida Sinica, vol. 28, pp. 277-284, 2015.

[75] S. Namilae and N. Chandra, "Role of atomic scale interfaces in the compressive behavior of carbon nanotubes in composites," Composites Science and Technology, vol. 66, pp. 2030-2038, 2006.

[76] D. D. T. K. Kulathunga and K. K. Ang, "Modeling and simulation of buckling of embedded carbon nanotubes," Computational Materials Science, vol. 81, pp. 233-238, 2014.

[77] H. Sun, "COMPASS: an ab initio force-field optimized for condensed-phase applications overview with details on alkane and benzene compounds," The Journal of Physical Chemistry B, vol. 102, pp. 7338-7364, 1998.

[78] D. W. Brenner, O. A. Shenderova, J. A. Harrison, S. J. Stuart, B. Ni, and S. B. Sinnott, "A second-generation reactive empirical bond order (REBO) potential energy expression for hydrocarbons," Journal of Physics: Condensed Matter, vol. 14, p. 783, 2002.

[79] S. Plimpton, "Fast parallel algorithms for short-range molecular dynamics," Journal of Computational Physics, vol. 117, pp. 1-19, 1995.

[80] P. Dauber-Osguthorpe, V. A. Roberts, D. J. Osguthorpe, J. Wolff, M. Genest, and A. T. Hagler, "Structure and energetics of ligand binding to proteins: Escherichia coli dihydrofolate reductase-trimethoprim, a drugreceptor system," Proteins: Structure, Function, and Bioinformatics, vol. 4, pp. 31-47, 1988.

[81] A. Alian, S. Kundalwal, and S. Meguid, "Multiscale modeling of carbon nanotube epoxy composites," Polymer, vol. 70, pp. 149-160, 2015.

[82] L. Martínez, R. Andrade, E. G. Birgin, and J. M. Martínez, "PACKMOL: A package for building initial configurations for molecular dynamics simulations," Journal of Computational Chemistry, vol. 30, pp. 21572164, 2009.

[83] W. Humphrey, A. Dalke, and K. Schulten, "VMD: Visual molecular dynamics," Journal of Molecular Graphics, vol. 14, pp. 33-38, 1996.

[84] Q. Wang, V. K. Varadan, Y. Xiang, Q. K. Han, and B. C. Wen, "On instability of single-walled carbon nanotubes with a vacancy defect," International Journal of Structural Stability and Dynamics, vol. 8, pp. 357-366, 2008.

[85] S. Nouranian, C. Jang, T. E. Lacy, S. R. Gwaltney, H. Toghiani, and C. U. Pittman Jr, "Molecular dynamics simulations of vinyl ester resin monomer interactions with a pristine vapor-grown carbon nanofiber and their implications for composite interphase formation," Carbon, vol. 49, pp. 3219-3232, 2011.

[86] C. Jang, S. Nouranian, T. E. Lacy, S. R. Gwaltney, H. Toghiani, and C. U. Pittman Jr, "Molecular dynamics simulations of oxidized vapor-grown carbon nanofiber surface interactions with vinyl ester resin monomers," Carbon, vol. 50, pp. 748-760, 2012. 
[87] C. Wang, Y. Zhang, Y. Xiang, and J. Reddy, "Recent studies on buckling of carbon nanotubes," Applied Mechanics Reviews, vol. 63, p. 030804, 2010.

[88] M. Bohlén and K. Bolton, "Molecular dynamics studies of the influence of single wall carbon nanotubes on the mechanical properties of Poly(vinylidene fluoride)," Computational Materials Science, vol. 68, pp. 73-80, 2013.

[89] S. C. Chowdhury and T. Okabe, "Computer simulation of carbon nanotube pull-out from polymer by the molecular dynamics method," Composites Part A-Applied Science and Manufacturing, vol. 38, pp. 747-754, 2007.

[90] L. G. Zhou and S. Q. Shi, "Adsorption of foreign atoms on Stone-Wales defects in carbon nanotube," Carbon, vol. 41, pp. 613-615, 2003.

[91] Y. Zhang, C. Wang, W. Duan, Y. Xiang, and Z. Zong, "Assessment of continuum mechanics models in predicting buckling strains of single-walled carbon nanotubes," Nanotechnology, vol. 20, p. 395707, 2009.

[92] Y. Zhang, V. Tan, and C. Wang, "Effect of chirality on buckling behavior of single-walled carbon nanotubes," Journal of Applied Physics, vol. 100, p. 074304, 2006. 